\title{
QUASISTATIC CRACK GROWTH BASED ON VISCOUS APPROXIMATION: A MODEL WITH BRANCHING AND KINKING
}

\author{
VITO CRISMALE AND GIULIANO LAZZARONI
}

\begin{abstract}
Employing the technique of vanishing viscosity and time rescaling, we show the existence of quasistatic evolutions of cracks in brittle materials in the setting of antiplane shear. The crack path is not prescribed a priori and is chosen in an admissible class of piecewise regular sets that allows for branching and kinking.
\end{abstract}

Keywords: brittle fracture, crack propagation, free-discontinuity problems, vanishing viscosity, local minimizers, Griffith's criterion, energy release rate.

2010 Mathematics Subject Classification: 35R35, 35Q74, 74R10, 74G70, 49J45.

\section{Contents}

1. Introduction

2. The admissible cracks

3. The time-incremental problems

4. Viscous evolutions

5. The vanishing viscosity limit

References

1
3
8
10
17
17

\section{INTRODUCTION}

The problem of the existence of quasistatic evolutions for Griffith's fracture [14] has been extensively studied in recent years in the mathematical community. (See [2] for an account on the literature and [23] for the relations with the abstract theory of rate-independent systems.) Nonetheless, the formulation of a realistic solution concept still deserves investigation.

The notion of globally stable evolution in the context of Griffith's fracture was proposed in [13] and then studied in several works, see e.g. [10, 3, 12, 8, 9], which provided a wide range of results under general assumptions on the energies and on the cracks. In this case, one selects configurations that are global minimizers of the stored elastic energy plus the energy dissipated in crack growth. However, this restriction produces unphysical effects such as early jumps in time [24].

On the other hand, propagation criteria based on local minimization $[26,15,17,19]$ are more satisfactory but require that the admissible cracks are regular in order to give mathematical sense to the energy release rate (which is the derivative of the elastic energy with respect to the elongation of the crack). In this context, the present paper is a contribution towards a more realistic description of quasistatic fracture that includes branching and kinking.

Local minimality can be enforced by employing the well established method of vanishing viscosity, first proposed in [11], then refined in [20, 21, 22], and applied e.g. in [7, 16, 6] for the analysis of plasticity and damage. Following this approach, a quasistatic evolution is obtained as a limit of solutions to rate-dependent systems that contain a viscous dissipation tending to zero. The viscous approximation of quasistatic crack growth was studied only when the deformations are functions of two variables and the cracks are represented as regular, one-dimensional sets. More precisely, in $[15,17]$ the crack evolves on a given, smooth curve, while in $[18,19]$ the crack is not prescribed a priori, but chosen in a class of admissible curves of class $C^{1,1}$.

In this work, in the setting of antiplane linearized elasticity we extend the previous results of [19] to a larger class $\mathcal{S}$ of cracks, already introduced in [27] to study viscous evolutions. The

Preprint SISSA 30/2016/MATE. 
admissible cracks in $\mathcal{S}$ may have many connected components, each of them being the union of a certain number of branches that are regular curves of the type considered in [19]. Moreover, some topological and geometrical restrictions are imposed in order to guarantee that $\mathcal{S}$ is closed with respect to the Hausdorff convergence, that the number of connected components and of branches is uniformly bounded in $\mathcal{S}$, and that the uncracked part of the body is always a connected set. These conditions allow for cracks displaying branching and kinking.

In order to show the existence of evolutions, we follow the strategy of $[15,19]$, based on time discretization. Specifically, for $k \in \mathbb{N}$ we introduce a subdivision of the time interval in equispaced $k+1$ nodes. At each time of the subdivision, we solve an incremental minimum problem where the competitors are all cracks in $\mathcal{S}$ that are larger than the crack found in the previous iteration. The functional to be minimized is the sum of three terms: the linearized elastic energy, the length of the crack (where the toughness is normalized to one), and a quadratic dissipation multiplied by $\varepsilon k$, where $\varepsilon>0$ is a viscous parameter. The quadratic dissipation depends on the elongation of the crack and results in a penalization of brutal crack propagation when $k$ is large.

We thus obtain a sequence of approximate discrete-time evolutions (piecewise constant in time), depending on the viscous parameter $\varepsilon$. We pass to the limit first as $k \rightarrow \infty$, obtaining evolutions continuous in time, and then as $\varepsilon \rightarrow 0$, obtaining a rate-independent evolution. The first passage to the limit was already studied in [27] in order to prove the existence of viscously regularized evolutions satisfying an energy inequality. In this paper, we complete the work initiated there by showing the energy-dissipation balance at viscous level and by passing to the limit as the viscous parameter tends to zero.

The evolution found in the limit as $\varepsilon \rightarrow 0$ satisfies the quasistatic energy-dissipation balance, as well as the Griffith principle. More precisely, the evolution has an at most countable number of time discontinuities. In the continuity set, the Griffith principle requires that

- the crack growth is irreversible, i.e., the crack is nondecreasing in time;

- the energy release rate (relative to each branch) never exceeds the toughness (here normalized to one);

- a branch can grow only if the corresponding energy release rate equals the toughness.

The quasistatic evolution found in this work satisfies the Griffith principle with the following limitation: the second law holds only when the branch tip does not meet a certain set of exceptional points. Such exceptional points are of two types: either they are points of branching or kinking, or they are points where the evolution stops because of the geometric restrictions on the cracks; moreover, also the limits of exceptional points of the approximating viscous evolutions have to be included among the exceptional points of the quasistatic evolution. Because of the restrictions on the class of admissible cracks, it turns out that the exceptional points are in a finite number. A full understanding of the Griffith principle at singular points would require to characterize the limit of the energy release rates of a sequence of irregular cracks converging in the sense of Hausdorff. However, the characterizations of the energy release rate of a crack at an irregular point given in $[25,4,5,1]$ do not provide the desired continuity properties.

For the passage to the limit as $\varepsilon \rightarrow 0$, we follow the strategy of $[11,15,7,19,20]$. We reparametrize the viscous evolutions in order to obtain a uniform bound on the time derivative of the crack length, thus as $\varepsilon \rightarrow 0$ we obtain an evolution continuous in time. In the reparametrized time scale, there is an at most countable number of intervals where the behavior is quasistatic and the Griffith principle holds (with the limitation described above). Each of the remaining (at most countable) intervals corresponds to a time discontinuity in the original time scale. The rescaled evolution is a countinuous interpolation of the quasistatic evolution. Whenever a branch shows a brutal propagation, the energy release rate (relative to that branch) is larger than or equal to the toughness in the time interval corresponding to the jump.

The structure of the paper is the following. In Section 2 we give the definition of the class of admissible cracks based on the one introduced in [27]; we prove some properties that come useful in the rest of the paper, in particular an estimate on the energy release rate. Section 3 contains the definition of the time-incremental problems and the statements of some results borrowed from [27]. In Section 4 we pass to the time-continuous limit as $k \rightarrow \infty$, obtaining a family of viscous evolutions; in particular we prove the viscous energy balance and further properties of the viscous solutions that are needed to pass to the limit as the viscous parameter $\varepsilon$ tends to zero. The latter passage to the limit is the subject of Section 5, where we study rescaled evolutions. 


\section{THE ADMISSIBLE CRACKS}

In the setting of antiplane elasticity, we consider a brittle body whose reference configuration is a cylinder $\Omega \times \mathbb{R} \subset \mathbb{R}^{3}$, with $\Omega \subset \mathbb{R}^{2}$ an open, bounded, connected, Lipschitz set. The deformations of the body are of the type

$$
\left(x_{1}, x_{2}, x_{3}\right) \mapsto\left(x_{1}, x_{2}, x_{3}+u\left(x_{1}, x_{2}\right)\right),
$$

where $u: \Omega \rightarrow \mathbb{R}$ is the corresponding displacement. We assume that the body can be fractured, that it has a perfectly elastic behaviour outside the cracked region, and that no force is transmitted across the crack. We now define the class of admissible cracks, denoted by $\mathcal{S}$, basing on the one introduced in [27]: this allows us to consider cracks with branching and kinking.

Starting from an initial fracture $\Gamma_{0} \in \mathcal{S}$, we study the evolution of cracks under the requirement that the displacement $u(t)$ is equal to a prescribed function $w(t)$ on the Dirichlet part of the boundary $\partial_{D} \Omega$, where $w \in A C\left([0, T] ; H^{1}(\Omega)\right)$. Given $t \in[0, T]$ and $\Gamma \in \mathcal{S}, u(t): \Omega \backslash \Gamma \rightarrow \mathbb{R}$ is the unique minimum point $u(w(t) ; \Gamma)$ of the elastic energy $\frac{1}{2}\|\nabla u\|_{2}^{2}$ under the condition $u=w(t)$ on $\partial_{D} \Omega$. The corresponding elastic energy associated to the crack $\Gamma$ and to the boundary displacement $w(t)$ is

$$
\mathcal{E}(w(t) ; \Gamma):=\min \left\{\frac{1}{2}\|\nabla u\|_{2}^{2}: u \in H^{1}(\Omega \backslash \Gamma), u=w(t) \text { on } \partial_{D} \Omega\right\}=\frac{1}{2}\|\nabla u(w(t) ; \Gamma)\|_{2}^{2} .
$$

In the framework of Griffith's theory [14], the energy dissipated to open a crack is proportional to the crack length. Normalizing the proportionality constant to 1, we define the total energy corresponding to $\Gamma$ and $w(t)$,

$$
\mathcal{F}(w(t) ; \Gamma):=\mathcal{E}(w(t) ; \Gamma)+\mathcal{H}^{1}(\Gamma)
$$

We now describe the class of admissible cracks $\mathcal{S}$ and its main properties, basing on [27]. Every admissible crack is the union of curves in the class $\mathcal{R}_{\eta}$, introduced in $[18,19]$, and here slightly modified. Henceforth, we will say that $\Gamma \subset \mathbb{R}^{2}$ is a simple curve of class $C^{1,1}$ if there is a parametrization $\gamma \in C^{1,1}\left([0, L] ; \mathbb{R}^{2}\right)$, for some $L>0$, such that $\gamma$ is injective and $\gamma([0, L])=\Gamma$.

Definition 2.1. Let $\eta>0$. Let $\Gamma \subset \mathbb{R}^{2}$ be a simple curve of class $C^{1,1}$ such that $\Omega \backslash \Gamma$ is open and connected. Given an arc-length parametrization of $\Gamma, \gamma:[0, L] \rightarrow \mathbb{R}^{2}$, we call $p_{1}:=\gamma(0)$ and $p_{2}:=\gamma(L)$ the endpoints of $\Gamma$. We say that $\Gamma \in \mathcal{R}_{\eta}$ if and only if

(a) $\mathcal{H}^{1}(\Gamma)>0$ and $\Gamma \subset \subset \Omega$;

(b) for every $x \in \Gamma$ there exist two open balls $B_{1}, B_{2}$ of radius $\eta$, such that

$$
\left(B_{1} \cup B_{2}\right) \cap(\Gamma \cup \partial \Omega)=\emptyset \quad \text { and } \quad \bar{B}_{1} \cap \bar{B}_{2}=\{x\} ;
$$

(c) we have that $\Gamma \cap\left(B_{\eta}\left(q_{1}\right) \cup B_{\eta}\left(q_{2}\right)\right)=\emptyset$, where

$$
q_{i}=p_{i}+\eta \frac{\dot{\gamma}\left(p_{i}\right)}{\left|\dot{\gamma}\left(p_{i}\right)\right|} \quad \text { for } i=1,2 .
$$

In order to account for branching and kinking, it is convenient to introduce two types of neighborhoods of a curve $\Gamma \in \mathcal{R}_{\eta}$. They depend on two parameters

$$
\beta \in(0, \eta / 3) \text { and } \theta \in(0, \pi / 4)
$$

fixed throughout the paper.

Let $\Gamma \in \mathcal{R}_{\eta}, \gamma:[0, L] \rightarrow \mathbb{R}^{2}$ be its arc-length parametrization, and $\dot{\gamma}(s)^{\perp}$ be normal to $\dot{\gamma}(s)$ with $\left|\dot{\gamma}(s)^{\perp}\right|=1$. We define

$$
\begin{aligned}
\mathcal{P}_{1}(\Gamma, p) & :=\left\{\gamma(s)+z \dot{\gamma}(s)^{\perp}: 0<s \leq L,|z|<\min \{s \tan \theta, \beta\}\right\} \\
& \cup\left\{\gamma(L)+(s-L) \dot{\gamma}(L)+z \dot{\gamma}(L)^{\perp}: L \leq s<L+\beta,|z|<\min \left\{s \tan \theta, \sqrt{\beta^{2}-(s-L)^{2}}\right\}\right\},
\end{aligned}
$$

where $p=\gamma(0)$, and

$$
\mathcal{P}_{2}(\Gamma):=\left\{\gamma(s)+z \dot{\gamma}(s)^{\perp}: 0<s<L,|z|<\min \{s \tan \theta, \beta,(L-s) \tan \theta\}\right\} .
$$

Notice that $\mathcal{P}_{1}(\Gamma, p)$ and $\mathcal{P}_{2}(\Gamma)$ are neighborhoods of $\gamma((0, L])$ and $\gamma((0, L))$, respectively. We refer to them as the 1-sided and the 2 -sided pencil-like neighborhoods of $\Gamma$, respectively. Moreover, two curves $\Gamma_{1}, \Gamma_{2} \in \mathcal{R}_{\eta}$ may intersect at most in the endpoints of $\Gamma_{1}$ if $\mathcal{P}_{2}\left(\Gamma_{1}\right) \cap \Gamma_{2}=\emptyset$, and at most in $p$ if $\mathcal{P}_{1}\left(\Gamma_{1}, p\right) \cap \Gamma_{2}=\emptyset$. See Figure 1 .

We introduce a class $\widehat{\mathcal{S}}$ of connected sets, that are union of elements of $\mathcal{R}_{\eta}$. 

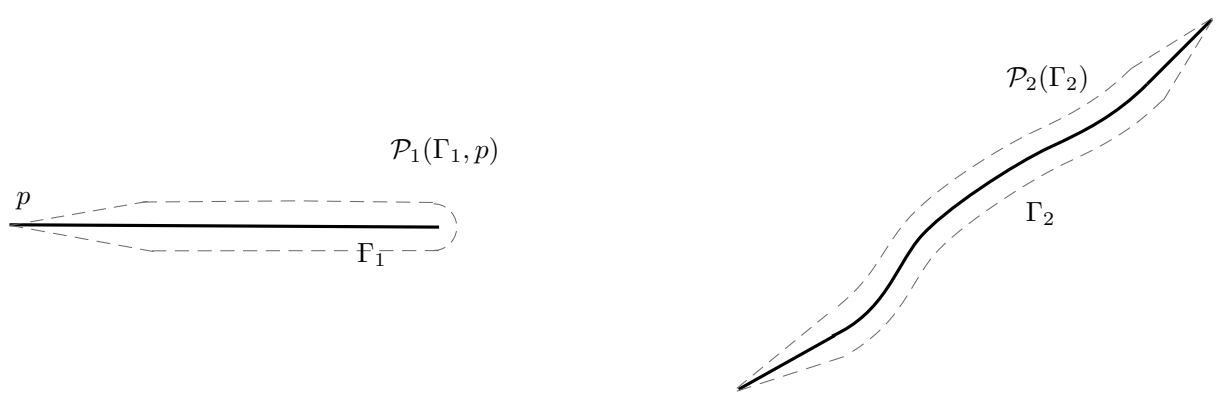

Figure 1: The pencil-like neighborhoods.

Definition 2.2. The class $\widehat{\mathcal{S}}$ is given by the connected sets $K \subset \mathbb{R}^{2}$ such that $\Omega \backslash K$ is open and connected,

with $m \in \mathbb{N}$, and $K$ has the form

$$
\mathcal{H}^{1}(K) \geq \frac{\beta}{\tan \theta}
$$

where the following hold:

$$
K=\bigcup_{j=1}^{m} \widetilde{K}_{j}
$$

(1) $\widetilde{K}_{j} \in \mathcal{R}_{\eta}$ for every $j$;

(2) if $\widetilde{K}_{i} \cap \widetilde{K}_{j} \neq \emptyset$ for $i \neq j$, then they intersect in one of their endpoints;

(3) if $\widetilde{K}_{i} \cup \widetilde{K}_{j} \in \mathcal{R}_{\eta}$, then there exists $\widetilde{K}_{l}, l \neq i, j$, such that $\widetilde{K}_{i} \cap \widetilde{K}_{j} \cap \widetilde{K}_{l} \neq \emptyset$;

(4) let $p_{0}, p_{1}$ be the endpoints of $\widetilde{K}_{j}$; if $p_{0} \in \widetilde{K}_{j} \cap \widetilde{K}_{l_{0}}$ for some $l_{0} \neq j$ and $p_{1} \notin \widetilde{K}_{l}$ for any $l \neq j$, then

$$
\mathcal{P}_{1}\left(\widetilde{K}_{j}, p_{0}\right) \cap \widetilde{K}_{l}=\emptyset \quad \text { for every } l \neq j ;
$$

(5) let $p_{0}, p_{1}$ be the endpoints of $\widetilde{K}_{j}$; if $p_{0} \in \widetilde{K}_{j} \cap \widetilde{K}_{l_{0}}$ and $p_{1} \in \widetilde{K}_{j} \cap \widetilde{K}_{l_{1}}$ for some $l_{0}, l_{1} \neq j$, then

$$
\mathcal{P}_{2}\left(\widetilde{K}_{j}\right) \cap \widetilde{K}_{l}=\emptyset \quad \text { for every } l \neq j
$$

We call any $\widetilde{K}_{j}$ a branch of $K$, and we define $I_{1}(K)$ and $I_{2}(K)$ as the sets of branches of $K$ satisfying the assumptions in (4) and (5), respectively.

Remark 2.3. It is possible to see that there exists a modulus of continuity $\omega$ (i.e., a continuous nondecreasing function $\omega:[0,+\infty) \rightarrow[0,+\infty)$ with $\omega(0)=0)$ such that the following holds: given $\Gamma \in \mathcal{R}_{\eta}$ and its arc-length parametrization $\gamma:[0, L] \rightarrow \mathbb{R}^{2}$

$$
\overline{B_{\omega(s)}(\gamma(s))} \subset \mathcal{P}_{1}(\Gamma, \gamma(0)) \text { and } \overline{B_{\omega(s) \wedge \omega(L-s)}(\gamma(s))} \subset \mathcal{P}_{2}(\Gamma) \text { for every } s \in(0, L) .
$$

For future convenience, without loss of generality we assume that $\omega(s)<s$ for $s>0$.

Every admissible crack is the union of sets $K$ as in Definition 2.2, with some geometric restrictions. See Figure 2.

Definition 2.4. Let $\Gamma$ be a set of the form

$$
\Gamma=\bigcup_{j=1}^{N} K_{j}
$$

with $K_{j} \in \widehat{\mathcal{S}}$ and $N \in \mathbb{N}$, and let us define

- the set of the special points of $\Gamma$

$\mathrm{S}_{\Gamma}:=\left\{x \in \Gamma: \exists v_{1}, v_{2} \in \mathbb{R}^{2}\right.$ unit vectors tangent to $\Gamma$ at $x$ s.t. $\left.v_{1} \cdot v_{2} \neq \pm 1\right\} ;$

- the set of the crack tip points of $\Gamma$

$\mathrm{T}_{\Gamma}:=\left\{x \in \Gamma: \exists r>0\right.$ s.t. $\Gamma \cap \overline{B_{r}(x)} \in \mathcal{R}_{\eta}$ and $x$ is an endpoint of $\left.\Gamma \cap \overline{B_{r}(x)}\right\} ;$

- the set of the regular points of $\Gamma$

$\mathrm{R}_{\Gamma}:=\Gamma \backslash\left(\mathrm{T}_{\Gamma} \cup \mathrm{S}_{\Gamma}\right)=\left\{x \in \Gamma: \exists r>0\right.$ s.t. $\Gamma \cap \overline{B_{r}(x)} \in \mathcal{R}_{\eta}$ with $x$ in the relative interior of $\left.\Gamma\right\}$. 


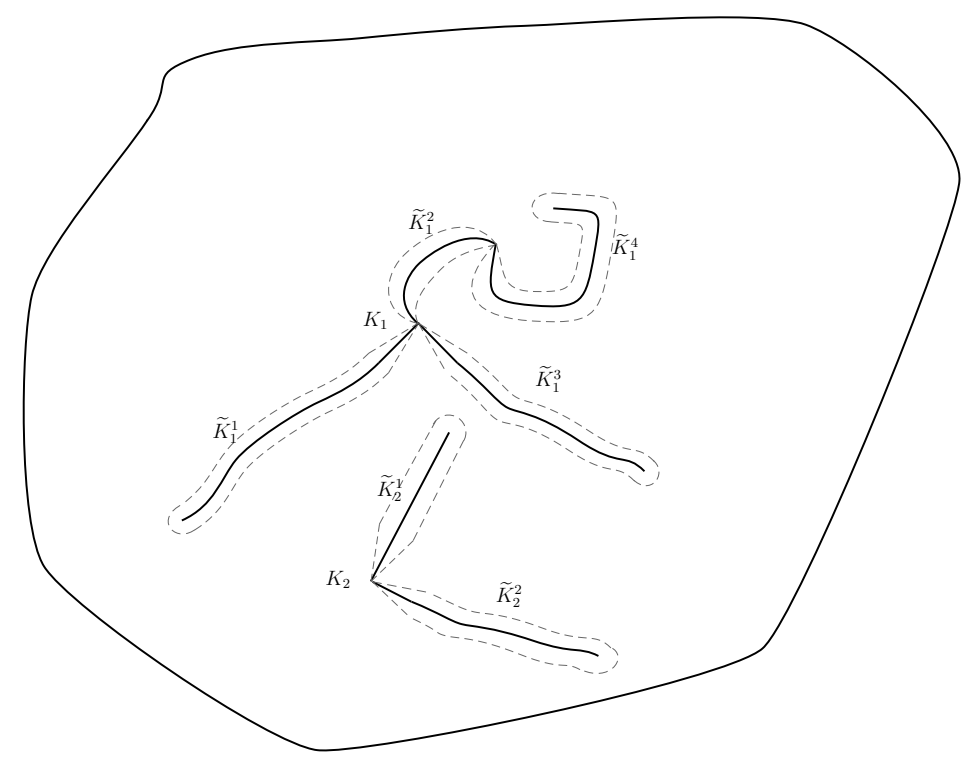

Figure 2: A crack $\Gamma \in \mathcal{S}$ with two connected components $K_{1}$ and $K_{2}$, with $K_{i}=\bigcup_{j} \widetilde{K}_{i}^{j}$. The pencil-like neighborhoods are delimited by dashed lines. Due to the kinked shape of the 2-sided (resp., 1-sided) pencil-like neighborhoods around both (resp., one of) the endpoints, the branching phenomenon is allowed, but there is a restriction on the number of branches. Moreover, the conditions (2) and (3) in Definition 2.2 describe a sort of "maximality" of each branch in the class $\mathcal{R}_{\eta}$ with respect to inclusion. Indeed, $\widetilde{K}_{1}^{2} \cup \widetilde{K}_{1}^{3} \in \mathcal{R}_{\eta}$ but we have two different branches $\widetilde{K}_{1}^{2}$ and $\widetilde{K}_{1}^{3}$ due to the presence of $\widetilde{K}_{1}^{1}$.

We say that $\Gamma \in \mathcal{S}$ if

(1) for every $j \in\{1, \ldots, N\}$,

if $K_{j} \in \mathcal{R}_{\eta}$, then $\mathrm{d}\left(K_{j}, K_{m}\right) \geq \beta$ for $m \neq j$;

if $\widetilde{K} \in I_{1}\left(K_{j}\right)$ and $p_{0}$ is its endpoint s.t. $p_{0} \in \mathrm{S}_{\Gamma}$, then $\mathcal{P}_{1}\left(\widetilde{K}, p_{0}\right) \cap K_{m}=\emptyset$ for $m \neq j$;

if $\widetilde{K} \in I_{2}\left(K_{j}\right)$, then $\mathcal{P}_{2}(\widetilde{K}) \cap K_{m}=\emptyset$ for $m \neq j$;

(2) for every $x_{1} \neq x_{2}$ in $\mathrm{S}_{\Gamma}$,

$$
\left|x_{1}-x_{2}\right| \geq \beta\left(\frac{2}{\tan \theta}+1\right) .
$$

It turns out that the sets $K_{j}$ as in (2.3) are the connected components of $\Gamma$. We further underline that, if $\widetilde{K} \in I_{1}\left(K_{j}\right)$, then one of its endpoints belongs to $\mathrm{S}_{\Gamma}$ and the other one to $\mathrm{T}_{\Gamma}$. Indeed, $\mathrm{T}_{\Gamma}$ consists of the endpoints of the type just described and of all the endpoints of the connected components of $\Gamma$ that belong to $\mathcal{R}_{\eta}$.

Notice that, for every $\Gamma \in \mathcal{S}, \Omega \backslash \Gamma$ is connected. Indeed, $\Omega \backslash K_{j}$ is connected for every connected component $K_{j}$ of $\Gamma$, by Definition 2.2, and the sets $K_{j}$ are pairwise disjoint, by conditions (2.4) and (2.5). On the other hand, if $\Omega \backslash \Gamma$ is connected, then $\Omega \backslash K$ is connected for every connected component $K$ of $\Gamma$.

Our definition of $\mathcal{S}$ is slightly different with respect to the one in [27]: indeed, we have prescribed that $\Omega \backslash \Gamma$ is connected, for every $\Gamma \in \mathcal{S}$. We then have to check that this further condition is preserved under Hausdorff convergence of curves in $\mathcal{S}$. See [27, Lemma 4.1] for similar properties.

Definition 2.5. Given two compact subsets $\Gamma, \Gamma^{\prime} \subset \bar{\Omega}$, their Hausdorff distance is given by

$$
\mathrm{d}_{H}\left(\Gamma^{\prime} ; \Gamma\right):=\max \left\{\sup _{x \in \Gamma^{\prime}} \operatorname{dist}(x, \Gamma), \sup _{x \in \Gamma} \operatorname{dist}\left(x, \Gamma^{\prime}\right)\right\},
$$

with the conventions $\operatorname{dist}(x, \emptyset)=\operatorname{diam} \Omega$ and $\sup \emptyset=0$. A sequence $\Gamma_{k}$ of compact subsets of $\bar{\Omega}$ converges to $\Gamma$ in the Hausdorff metric if $\mathrm{d}_{H}\left(\Gamma_{k} ; \Gamma\right) \rightarrow 0$. In this case we write $\Gamma_{k} \stackrel{\mathcal{H}}{\longrightarrow} \Gamma$.

Proposition 2.6. Let $\Gamma_{k} \in \mathcal{S}$ be such that $\Gamma_{k} \stackrel{\mathcal{H}}{\longrightarrow} \Gamma$. Then $\Omega \backslash \Gamma$ is connected. 
Proof. We first observe that, by Definition 2.1 and [18, Proposition 2.9], the class $\mathcal{R}_{\eta}$ is closed. Therefore if $\Gamma_{k} \in \mathcal{R}_{\eta}$ for every $k$, then $\Omega \backslash \Gamma$ is connected. Moreover, by [27, Lemma 3.9], the total number of branches of $\Gamma_{k}$ is equibounded in $k$ (see Definition 2.2 for the definition of branches).

By contradiction, assume that there exists an open connected set $\Omega^{\prime} \subset \subset \Omega$ such that $\partial \Omega^{\prime} \subset \Gamma$. Then there exist $x \in \partial \Omega^{\prime}, \widetilde{K}_{k}^{1}, \widetilde{K}_{k}^{2}$ different branches of $\Gamma_{k}$, and $x_{k}^{1} \in \widetilde{K}_{k}^{1}, x_{k}^{2} \in \widetilde{K}_{k}^{2}$, such that

$$
x_{k}^{1}, x_{k}^{2} \rightarrow x
$$

and $\mathcal{H}^{1}\left(\widetilde{K}_{k}^{1}\right)>C_{0}, \mathcal{H}^{1}\left(\widetilde{K}_{k}^{2}\right)>C_{0}$, for a positive constant $C_{0}$ independent of $k$. Since $\Omega^{\prime}$ is open and connected, we may assume that there exists $C_{1}>0$, independent of $k$, such that

$$
\mathrm{d}\left(x, \widetilde{K}_{k}^{1} \cap \widetilde{K}_{k}^{2}\right) \geq C_{1} .
$$

Notice that either $\widetilde{K}_{k}^{1} \cap \widetilde{K}_{k}^{2}$ is empty, or it contains only one point, which belongs to $\mathrm{S}_{\Gamma_{k}}$. In particular $x_{k}^{1} \neq x_{k}^{2}$.

We claim that, up to subsequences, there exists a positive constant $C_{2}$ such that

$$
\mathrm{d}\left(x_{k}^{1}, \mathrm{~S}_{\Gamma_{k}} \cap \widetilde{K}_{k}^{1}\right) \geq C_{2} \quad \text { or } \quad \mathrm{d}\left(x_{k}^{2}, \mathrm{~S}_{\Gamma_{k}} \cap \widetilde{K}_{k}^{2}\right) \geq C_{2} .
$$

Indeed, by contradiction, let $\mathrm{d}\left(x_{k}^{i}, \mathrm{~S}_{\Gamma_{k}} \cap \widetilde{K}_{k}^{i}\right) \rightarrow 0$ for $i=1,2$, and let $y_{k}^{i} \in \mathrm{S}_{\Gamma_{k}} \cap \widetilde{K}_{k}^{i}$ with $\left|x_{k}^{i}-y_{k}^{i}\right|=\mathrm{d}\left(x_{k}^{i}, \mathrm{~S}_{\Gamma_{k}} \cap \widetilde{K}_{k}^{i}\right)$. Notice that $y_{k}^{1}, y_{k}^{2} \rightarrow x$. If $y_{k}^{1}=y_{k}^{2}=: y_{k}$, then $y_{k} \in \widetilde{K}_{k}^{1} \cap \widetilde{K}_{k}^{2}$ and $y_{k} \rightarrow x$, in contradiction with (2.7). On the other hand, if $y_{k}^{1} \neq y_{k}^{2}$, we have that (2.5) is contradicted, since $\left|y_{k}^{1}-y_{k}^{2}\right| \rightarrow 0$. Then (2.8) is proved.

Assume that $x_{k}^{1}$ and $\widetilde{K}_{k}^{1}$ satisfy (2.8), and let $\gamma_{k}^{1}$ be an arc-length parametrization of $\widetilde{K}_{k}^{1}$. By Remark 2.3, we have that $\mathrm{d}\left(x_{k}^{1}, \widetilde{K}_{k}^{\prime}\right) \geq \omega\left(C_{2}\right)$ for any branch $\widetilde{K}_{k}^{\prime}$ of $\Gamma_{k}$ different from $\widetilde{K}_{k}^{1}$. In particular,

$$
\left|x_{k}^{1}-x_{k}^{2}\right| \geq \omega\left(C_{2}\right)
$$

for every $k$, in contradiction with (2.6). Therefore the result is proved.

In the following proposition we collect the most important properties of the class of admissible cracks. These can be proved following the same arguments as in [27]. The property of compactness of $\mathcal{S}$ with respect to the Hausdorff convergence employs Proposition 2.6.

Proposition 2.7. The class $\mathcal{S}$ introduced in Definition 2.4 is compact with respect to the Hausdorff convergence, and the length of the admissible cracks is uniformly bounded, as well as the number of the branches, of the singular points and of the tip points. In particular, for every $\Gamma \in \mathcal{S}, \Omega \backslash \Gamma$ is the union of a uniformly bounded number of Lipschitz open sets that intersect $\partial_{D} \Omega$.

Moreover, if $\Gamma_{k} \in \mathcal{S}$ are such that $\Gamma_{k} \stackrel{\mathcal{H}}{\longrightarrow} \Gamma$, then

(i) $\mathcal{H}^{1}\left(\Gamma_{k}\right) \rightarrow \mathcal{H}^{1}(\Gamma)$;

(ii) for every $p \in \mathrm{T}_{\Gamma}$ there exists a sequence $\left(p_{k}\right)_{k}$ with $p_{k} \in \mathrm{T}_{\Gamma_{k}}$ such that $p_{k} \rightarrow p$;

(iii) if $p_{k}^{1}, p_{k}^{2} \in \mathrm{T}_{\Gamma_{k}}, p_{k}^{1} \neq p_{k}^{2}$ and $\left(p_{k}^{1}\right)_{k},\left(p_{k}^{2}\right)_{k}$ are converging to $p \in \mathrm{T}_{\Gamma}$, then there exists a sequence $\left(y_{k}\right)_{k}$, with $y_{k} \in \mathrm{S}_{\Gamma_{k}}$, converging to $p$.

We can follow the arguments of [18] in order to define the notion of energy release rate relative to a crack tip. First, let us introduce the extensions of a crack near a tip. In the following discussion, we fix $\Gamma \in \mathcal{S}, p \in \mathrm{T}_{\Gamma}$, and $r>0$ as in the definition of crack tip.

Definition 2.8. We call extension of $\Gamma$ at $p$ any $\widetilde{\Gamma} \in \mathcal{S}$ such that $\Gamma \subsetneq \widetilde{\Gamma}$,

$$
\widetilde{\Gamma} \backslash \Gamma \subset \subset B_{r}(p) \text { and } \widetilde{\Gamma} \cap \overline{B_{r}(p)} \in \mathcal{R}_{\eta} \quad \text { for some } r .
$$

Remark 2.9. In the general case, there could exist points $p$ such that there are not extensions of $\Gamma$ at $p$. We denote

$$
\mathrm{G}_{\Gamma}:=\left\{p \in \mathrm{T}_{\Gamma}: \text { there are extensions of } \Gamma \text { at } p\right\} .
$$

If $\widetilde{\Gamma}$ is an extension of $\Gamma$ at $p$, let $L:=\mathcal{H}^{1}(\widetilde{\Gamma} \backslash \Gamma)$ and let $\widetilde{\gamma}_{p}:[0, L] \rightarrow \Omega$ be the arc-length parametrization of $(\widetilde{\Gamma} \backslash \Gamma) \cup\{p\}$ such that $\widetilde{\gamma}_{p}(0)=p$. Then

$$
(0, L] \ni s \mapsto \widetilde{\Gamma}_{s}^{p}:=\Gamma \cup \widetilde{\gamma}_{p}((0, s])
$$

is a family of extensions of $\Gamma$ in $p$ such that $\mathcal{H}^{1}\left(\widetilde{\Gamma}_{s}^{p} \backslash \Gamma\right)=s$. 
We also use the following notation:

$$
\Gamma_{p, r}:=\Gamma \cap \overline{B_{r}(p)} \in \mathcal{R}_{\eta} \quad \text { with } \quad \Gamma_{p, r} \cap \partial B_{r}(p)=:\{q\} .
$$

Let $p \in \mathrm{G}_{\Gamma}$ and let $\widetilde{\Gamma}$ be an extension of $\Gamma$ in $p$. It holds that

$$
\widetilde{\Gamma}_{p, r}:=\widetilde{\Gamma} \cap \overline{B_{r}(p)} \in \mathcal{R}_{\eta} \quad \text { with } \quad \widetilde{\Gamma}_{p, r} \cap \partial B_{r}(p)=\{q\} .
$$

Let $\widetilde{\gamma}_{p, r}:\left[0, l\left(\widetilde{\Gamma}_{p, r}\right)\right] \rightarrow \Omega$ be the arc-length parametrization of $\widetilde{\Gamma}_{p, r}$ such that $\gamma_{p, r}^{\prime}(0)=q$. As showed in [18], the function

$$
l \mapsto \mathcal{E}\left(w(t) ; \Gamma \cup \widetilde{\gamma}_{p, r}([0, l])\right)
$$

is differentiable at $l=\mathcal{H}^{1}\left(\Gamma_{p, r}\right)$ and the value of the derivative is independent of the choice of the extension $\widetilde{\Gamma}$. In order to see these properties, one employs the Poincaré inequality in $\Omega \backslash\left(\Gamma \cup \widetilde{\Gamma}_{p, r}\right)$, which holds since for every $\Gamma \subset \mathcal{S}, \Omega \backslash \Gamma$ is the union of a fixed number of Lipschitz open sets that intersect $\partial_{D} \Omega$. Then the following definition is well posed.

Definition 2.10. Let $p \in \mathrm{G}_{\Gamma}$. The energy release rate relative to $w(t), p$, and $\Gamma$ is

$$
\mathcal{G}(w(t) ; \Gamma, p):=-\left.\partial_{l} \mathcal{E}\left(w(t) ; \Gamma \cup \widetilde{\gamma}_{p, r}([0, l])\right)\right|_{l=\mathcal{H}^{1}\left(\Gamma_{p, r}\right)} .
$$

Notation. In the Sections $3,4,5$ we will use for every $t \in[0, T]$ the notation $\mathcal{E}(t ; \Gamma), \mathcal{F}(t ; \Gamma)$, and $\mathcal{G}(t ; \Gamma, p)$ respectively for $\mathcal{E}(w(t) ; \Gamma), \mathcal{F}(w(t) ; \Gamma)$, and $\mathcal{G}(w(t) ; \Gamma, p)$.

The following integral representation was proven in [18, Propositions 2.2 and 2.4].

Proposition 2.11. Let $\Gamma \in \mathcal{S}, p \in \mathrm{G}_{\Gamma}$ and $r>0$ such that

$$
\Gamma \cap \overline{B_{r}(p)} \in \mathcal{R}_{\eta} \text {. }
$$

Let $\gamma$ be the arc-length parametrization of $\Gamma \cap \overline{B_{r}(p)} \in \mathcal{R}_{\eta}$ with $p=\gamma(L), L=\mathcal{H}^{1}\left(\Gamma \cap \overline{B_{r}(p)}\right)$. Then

$$
\mathcal{G}(g ; \Gamma, p)=\int_{\Omega \backslash \Gamma}\left[\frac{\left(D_{1} u\right)^{2}-\left(D_{2} u\right)^{2}}{2}\left(D_{1} V^{1}-D_{2} V^{2}\right)+D_{1} u D_{2} u\left(D_{2} V^{1}+D_{1} V^{2}\right)\right] \mathrm{d} x,
$$

where $V$ is any vector field of class $C^{0,1}$ with compact support in $\Omega$ such that $V(\gamma(s))=\dot{\gamma}(s)$ for $s$ in a neighborhood of $L$, and $u=u(g ; \Gamma)$ is the unique minimum point of the elastic energy with boundary condition $g$ on $\partial_{D} \Omega$.

The integral representation allows us to deduce the fundamental continuity properties of the energy release rate with respect to the convergence of the curves, of the tips, and of the boundary displacements, provided that condition (2.10) holds uniformly.

Proposition 2.12. Let $\Gamma_{0}, \Gamma_{k}, \Gamma \in \mathcal{S}$ with $\Gamma_{0} \subset \Gamma_{k}, \Gamma_{0} \subset \Gamma$. Moreover, let $g_{k} \rightarrow g$ in $H^{1}\left(\Omega \backslash \Gamma_{0}\right)$ and $p_{k} \in \mathrm{G}_{\Gamma_{k}}, p \in \mathrm{G}_{\Gamma}$.

Assume that $\Gamma_{k} \stackrel{\mathcal{H}}{\longrightarrow} \Gamma, p_{k} \rightarrow p$, and that there exists $r>0$ such that

$$
\Gamma_{k} \cap \overline{B_{r}(p)} \in \mathcal{R}_{\eta} .
$$

Then

$$
\mathcal{G}\left(g_{k} ; \Gamma_{k}, p_{k}\right) \rightarrow \mathcal{G}(g ; \Gamma, p)
$$

and there exists a positive constant $C(\eta, r)$, where $\eta$ and $r$ are as in (2.12), such that

$$
\mathcal{G}\left(g_{k} ; \Gamma_{k}, p_{k}\right) \leq C(\eta, r) \sup _{k}\left\|\nabla u_{k}\right\|_{2}^{2} .
$$

Proof. Since $\Gamma_{k} \stackrel{\mathcal{H}}{\longrightarrow} \Gamma$ and the class $\mathcal{R}_{\eta}$ is closed with respect to Hausdorff convergence, we get that $\Gamma_{k} \cap \overline{B_{r}(p)} \stackrel{\mathcal{H}}{\longrightarrow} \Gamma \cap \overline{B_{r}(p)}$ and $\Gamma \cap \overline{B_{r}(p)} \in \mathcal{R}_{\eta}$.

Following the lines of [18, Theorem 2.12] and [27, Lemma 8.2], we extend $\Gamma_{k} \cap \overline{B_{r}(p)}$, for every $k$, and $\Gamma \cap \overline{B_{r}(p)}$ with a segment following the tangent direction to the curve at the tips $p_{k}$ and $p$. By the Implicit Function Theorem, there exist a neighborhood $U \subset B_{r}(p)$ of $p$ and two suitable coordinate axes such that the extended curves are parametrized in $U$ by $\left(x_{1}, \varphi_{k}\left(x_{1}\right)\right)$ and $\left(x_{1}, \varphi\left(x_{1}\right)\right)$, with $\varphi_{k}, \varphi$ of class $C^{1,1}$. Notice that, by definition of $\mathcal{R}_{\eta}$, we can take $U=B_{\eta \wedge r}(p)$. Indeed, if $K \in \mathcal{R}_{\eta}$ and $B$ is a ball of radius $\eta$, there are at most two points of $K$ such that the tangent vectors to $K$ at these points are orthogonal.

We now set

$$
V_{k}(x):=\zeta(x)\left(1, \dot{\varphi}_{k}\left(x_{1}\right)\right), \quad V(x):=\zeta(x)\left(1, \dot{\varphi}\left(x_{1}\right)\right)
$$


with $\zeta$ a cutoff function supported in $U$. Thus, by (2.11)

$$
\mathcal{G}\left(g_{k} ; \Gamma_{k}, p_{k}\right)=\int_{\Omega \backslash \Gamma_{k}}\left[\frac{\left(D_{1} u_{k}\right)^{2}-\left(D_{2} u_{k}\right)^{2}}{2}\left(D_{1} V_{k}^{1}-D_{2} V_{k}^{2}\right)+D_{1} u_{k} D_{2} u_{k}\left(D_{2} V_{k}^{1}+D_{1} V_{k}^{2}\right)\right] \mathrm{d} x,
$$

with $u_{k}:=u\left(g_{k} ; \Gamma_{k}\right)$, and an analogous identity holds for $\mathcal{G}(g ; \Gamma, p)$.

Since $\Gamma_{k} \cap \overline{B_{r}(p)} \stackrel{\mathcal{H}}{\longrightarrow} \Gamma \cap \overline{B_{r}(p)}$ and these are elements of $\mathcal{R}_{\eta}$, we get that

$$
\nabla V_{k} \stackrel{*}{\rightarrow} \nabla V \quad \text { in } L^{\infty}\left(\Omega ; \mathbb{R}^{2 \times 2}\right) .
$$

Notice that there exists a positive constant $C$, depending only on $\eta$ and $r$, such that

$$
\left|\nabla V_{k}\right| \leq C
$$

because $\ddot{\gamma}(s)$ is bounded by $\frac{1}{\eta}$ and $\nabla \zeta$ is controlled in terms of $r$ and $\eta$, since $U=B_{\eta \wedge r}(p)$.

By [10, Theorem 5.1] and the Poincaré inequality, $\nabla u_{k} \rightarrow \nabla u$ in $L^{2}\left(\Omega ; \mathbb{R}^{2}\right)$. Therefore we can pass to the limit in the identity above as $k \rightarrow \infty$. The inequality (2.13) follows from (2.14).

\section{THE TIME-INCREMENTAL PROBLEMS}

In this section we recall the construction of discrete-time approximated evolutions of viscous type, already presented in [27]. We fix a subdivision of the time interval in $k+1$ equispaced nodes and a viscosity parameter $\varepsilon>0$, and we solve incremental minimum problems on the class $\mathcal{S}$, thus allowing for new branches and kinks. The results in [27, Section 4] provide some a priori estimates, useful in order to pass to the limit as $k \rightarrow \infty$ to continuous-time viscous evolutions, and a discrete Griffith principle. In Section 4 we show new results on the viscous solutions, which permit to pass to the limit as $\varepsilon \rightarrow 0$ in Section 5 .

We fix a sequence of subdivisions of the interval $[0, T]$ consisting of equispaced nodes $\left(t_{k}^{i}\right)_{0 \leq i \leq k}$,

$$
t_{k}^{i}:=\frac{i}{k} T \text {. }
$$

We put $\tau:=\frac{1}{k}$ and we define

$$
\Gamma_{\varepsilon, k}^{0}:=\Gamma_{0} \in \mathcal{S}, \quad u_{\varepsilon, k}^{0}:=u\left(w(0) ; \Gamma_{0}\right) \equiv u_{0},
$$

and $\left(\Gamma_{\varepsilon, k}^{i}, u_{\varepsilon, k}^{i}\right)$ as a solution to the minimum problem

$$
\min \left\{\frac{1}{2}\|\nabla u\|_{2}^{2}+\mathcal{H}^{1}(\Gamma)+\frac{\varepsilon}{2 \tau} \sum_{\mathfrak{c} \in \mathcal{C}\left(\Gamma_{\varepsilon, k}^{i-1}, \Gamma\right)} \mathcal{H}^{1}(\mathfrak{c})^{2}: \Gamma \in \mathcal{S}, \Gamma \supset \Gamma_{\varepsilon, k}^{i-1}, u \in H^{1}(\Omega \backslash \Gamma), u=w(t) \text { on } \partial_{D} \Omega\right\},
$$

where $\mathcal{C}\left(\Gamma_{1}, \Gamma_{2}\right)$ is the set of the connected components of $\Gamma_{2} \backslash \Gamma_{1}$ for $\Gamma_{1} \subset \Gamma_{2} \in \mathcal{S}$. Equivalently one can define $\Gamma_{\varepsilon, k}^{i}$ as a solution to

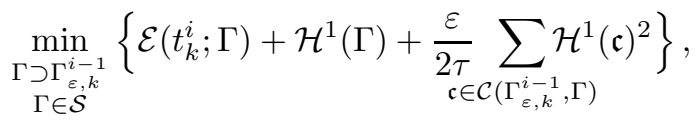

and

$$
u_{\varepsilon, k}^{i}:=u\left(w\left(t_{k}^{i}\right) ; \Gamma_{\varepsilon, k}^{i}\right) .
$$

By [27, Lemma 4.1 and Proposition 4.2] (recall also Proposition 2.7), problem (3.3) has a solution. Let us define the piecewise constant interpolations

$$
u_{\varepsilon, k}(t):=u_{\varepsilon, k}^{i}, \quad \Gamma_{\varepsilon, k}(t):=\Gamma_{\varepsilon, k}^{i}, \quad \underline{l}_{\varepsilon, k}(t):=\mathcal{H}^{1}\left(\Gamma_{\varepsilon, k}^{i}\right) \quad \text { for } t \in\left[t_{k}^{i}, t_{k}^{i+1}\right),
$$

and the piecewise affine interpolation

$$
l_{\varepsilon, k}(t):=\mathcal{H}^{1}\left(\Gamma_{\varepsilon, k}^{i}\right)+\frac{t-t_{k}^{i}}{\tau} \mathcal{H}^{1}\left(\Gamma_{\varepsilon, k}^{i+1} \backslash \Gamma_{\varepsilon, k}^{i}\right) \quad \text { for } t \in\left[t_{k}^{i}, t_{k}^{i+1}\right),
$$

with $u_{\varepsilon, k}(T):=u_{\varepsilon, k}^{k}, \Gamma_{\varepsilon, k}(T):=\Gamma_{\varepsilon, k}^{k}$, and $l_{\varepsilon, k}(T)=\underline{l}_{\varepsilon, k}(T):=\mathcal{H}^{1}\left(\Gamma_{\varepsilon, k}(T)\right)$. Let us set also

$$
\mathrm{T}_{\varepsilon, k}(t):=\mathrm{T}_{\Gamma_{\varepsilon, k}(t)}, \quad \mathrm{S}_{\varepsilon, k}(t):=\mathrm{S}_{\Gamma_{\varepsilon, k}(t)}, \quad \mathrm{R}_{\varepsilon, k}(t):=\mathrm{R}_{\Gamma_{\varepsilon, k}(t)}, \quad \mathrm{G}_{\varepsilon, k}(t):=\mathrm{G}_{\Gamma_{\varepsilon, k}(t)} .
$$

As usual, a priori bounds are derived by comparing the minimum value of the functional in (3.2) with the one assumed for the admissible pair $\left(\Gamma_{\varepsilon, k}^{i-1}, u_{\varepsilon, k}^{i-1}+w\left(t_{k}^{i}\right)-w\left(t_{k}^{i-1}\right)\right)$. By standard computations, and recalling that the number of connected components of curves in $\mathcal{S}$ is uniformly bounded, one gets the following estimates. 
Proposition 3.1. For every $\varepsilon, k$, and $t \in\left[t_{k}^{i}, t_{k}^{i+1}\right)$,

$$
\begin{gathered}
\mathcal{E}\left(t_{k}^{i} ; \Gamma_{\varepsilon, k}(t)\right)+\mathcal{H}^{1}\left(\Gamma_{\varepsilon, k}(t)\right)+\frac{\varepsilon}{2 \tau} \sum_{j=1}^{i}\left[\sum_{\mathfrak{c} \in \mathcal{C}\left(\Gamma_{\varepsilon, k}^{j-1}, \Gamma_{\varepsilon, k}^{j}\right)} \mathcal{H}^{1}(\mathfrak{c})^{2}\right] \\
\leq \mathcal{E}\left(0 ; \Gamma_{0}\right)+l_{0}+\int_{0}^{t_{k}^{i}}\left\langle\nabla u_{\varepsilon, k}(s), \nabla \dot{w}(s)\right\rangle \mathrm{d} s+\delta(k),
\end{gathered}
$$

where

$$
\delta(k)=\sup _{1 \leq i \leq k}\left(\int_{t_{k}^{i}-1}^{t_{k}^{i}}\|\nabla \dot{w}(s)\|_{2} \mathrm{~d} s\right) \int_{0}^{T}\|\nabla \dot{w}(s)\|_{2} \mathrm{~d} s \rightarrow 0 \quad \text { as } k \rightarrow \infty .
$$

Moreover, there exists a constant $\bar{C}>0$, independent of $\varepsilon, k$, and $t$, such that

$$
\mathcal{E}\left(t ; \Gamma_{\varepsilon, k}(t)\right)+\mathcal{H}^{1}\left(\Gamma_{\varepsilon, k}(t)\right) \leq \bar{C}, \quad \frac{\varepsilon}{\tau} \sum_{j=1}^{i}\left[\sum_{\mathfrak{c} \in \mathcal{C}(j-1, j)} \mathcal{H}^{1}(\mathfrak{c})^{2}\right] \leq \bar{C}, \quad \varepsilon\left\|l_{\varepsilon, k}\right\|_{H^{1}(0, T)} \leq \bar{C},
$$

where $\mathcal{C}(j-1, j)=\mathcal{C}\left(\Gamma_{\varepsilon, k}^{j-1}, \Gamma_{\varepsilon, k}^{j}\right)$.

We now introduce the notion of discrete velocity, for those tips in $\mathrm{T}_{\varepsilon, k}\left(t_{k}^{i}\right)$ such that the corresponding connected component of $\Gamma_{\varepsilon, k}^{i} \backslash \Gamma_{\varepsilon, k}^{i-1}$ does not contain elements of $\mathrm{S}_{\varepsilon, k}\left(t_{k}^{i}\right)$.

Definition 3.2. Let $t \in\left[t_{k}^{i}, t_{k}^{i+1}\right), p \in \mathrm{T}_{\varepsilon, k}(t)=\mathrm{T}_{\varepsilon, k}\left(t_{k}^{i}\right)$. If $p \in \mathrm{T}_{\varepsilon, k}\left(t_{k}^{i}\right) \cap \mathrm{T}_{\varepsilon, k}\left(t_{k}^{i-1}\right)$, we set

$$
v_{\varepsilon, k}(t, p):=0 \text {. }
$$

Otherwise let $\mathfrak{c}_{\varepsilon, k}^{p} \in \mathcal{C}\left(\Gamma_{\varepsilon, k}^{i-1}, \Gamma_{\varepsilon, k}^{i}\right)$ be such that $p \in \mathfrak{c}_{\varepsilon, k}^{p}$. If $\left[\mathfrak{c}_{\varepsilon, k}^{p} \backslash \Gamma_{\varepsilon, k}^{i-1}\right] \cap \mathrm{S}_{\varepsilon, k}\left(t_{k}^{i}\right)=\emptyset$, we set

$$
v_{\varepsilon, k}(t, p):=\frac{\mathcal{H}^{1}\left(\mathfrak{c}_{\varepsilon, k}^{p}\right)}{\tau} .
$$

The following result is the discrete version of the Griffith principle.

Proposition 3.3. Let $t$ and $p \in \mathrm{G}_{\varepsilon, k}(t)$ be such that $v_{\varepsilon, k}(t, p)$ is defined as in Definition 3.2. Then

$$
\begin{aligned}
& v_{\varepsilon, k}(t, p) \geq 0, \\
& \mathcal{G}\left(t_{k}^{i} ; \Gamma_{\varepsilon, k}^{i}, p\right) \leq 1+\varepsilon v_{\varepsilon, k}(t, p), \\
& {\left[-\mathcal{G}\left(t_{k}^{i} ; \Gamma_{\varepsilon, k}^{i}, p\right)+1+\varepsilon v_{\varepsilon, k}(t, p)\right] v_{\varepsilon, k}(t, p)=0 .}
\end{aligned}
$$

Proof. Property (3.5a) is trivial. By (3.3)

$$
\begin{gathered}
\mathcal{E}\left(t_{k}^{i} ; \Gamma_{\varepsilon, k}^{i}\right)+\mathcal{H}^{1}\left(\Gamma_{\varepsilon, k}^{i}\right)+\frac{\varepsilon}{2 \tau} \sum_{\mathfrak{c} \in \mathcal{C}\left(\Gamma_{\varepsilon, k}^{i-1}, \Gamma_{\varepsilon, k}^{i}\right)} \mathcal{H}^{1}(\mathfrak{c})^{2} \\
\leq \mathcal{E}\left(t_{k}^{i} ; \widetilde{\Gamma}_{s}^{p}\right)+\mathcal{H}^{1}\left(\widetilde{\Gamma}_{s}^{p}\right)+\frac{\varepsilon}{2 \tau} \sum_{\substack{\mathfrak{c} \in \mathcal{C}\left(\Gamma_{\varepsilon, k}^{i-1}, \widetilde{\Gamma}_{s}^{p}\right)}} \mathcal{H}^{1}(\mathfrak{c})^{2},
\end{gathered}
$$

where $\widetilde{\Gamma}_{s}^{p}$ is an extension of $\Gamma_{\varepsilon, k}^{i}$ in $p$ (recall Definition 2.8) such that

$$
\mathcal{H}^{1}\left(\widetilde{\Gamma}_{s}^{p} \backslash \Gamma_{\varepsilon, k}^{i}\right)=s .
$$

Dividing (3.6) by $s$ and letting $s \rightarrow 0$, by definition of energy release rate at $p$ and by (3.7) we obtain $(3.5 \mathrm{~b})$.

As for $(3.5 \mathrm{c})$, we can assume that $v_{\varepsilon, k}(t, p)>0$, and then that $\mathcal{H}^{1}\left(\mathfrak{c}_{\varepsilon, k}^{p}\right)>0$, for $\mathfrak{c}_{\varepsilon, k}^{p}$ as in Definition 3.2. Let us consider $0<s<\mathcal{H}^{1}\left(\mathfrak{c}_{\varepsilon, k}^{p}\right)$ and let $\widehat{\Gamma}_{s}^{p}$ be such that

$$
\Gamma_{\varepsilon, k}^{i} \backslash \mathfrak{c}_{\varepsilon, k}^{p} \subset \widehat{\Gamma}_{s}^{p} \subset \Gamma_{\varepsilon, k}^{i} \quad \text { and } \quad \mathcal{H}^{1}\left(\Gamma_{\varepsilon, k}^{i} \backslash \widehat{\Gamma}_{s}^{p}\right)=s
$$

This is a competitor for the minimum problem (3.3), and then

$$
\begin{aligned}
& \mathcal{E}\left(t_{k}^{i} ; \Gamma_{\varepsilon, k}^{i}\right)+\mathcal{H}^{1}\left(\Gamma_{\varepsilon, k}^{i}\right)+\frac{\varepsilon}{2 \tau} \sum_{\mathfrak{c} \in \mathcal{C}\left(\Gamma_{\varepsilon, k}^{i-1}, \Gamma_{\varepsilon, k}^{i}\right)} \mathcal{H}^{1}(\mathfrak{c})^{2} \\
& \quad \leq \mathcal{E}\left(t_{k}^{i} ; \widehat{\Gamma}_{s}^{p}\right)+\mathcal{H}^{1}\left(\widehat{\Gamma}_{s}^{p}\right)+\frac{\varepsilon}{2 \tau} \sum_{\mathfrak{c} \in \mathcal{C}\left(\Gamma_{\varepsilon, k}^{i-1}, \widehat{\Gamma}_{s}^{p}\right)} \mathcal{H}^{1}(\mathfrak{c})^{2} .
\end{aligned}
$$


Dividing by $s$ and letting $s \rightarrow 0$ we get (3.5c). Notice that $\mathcal{G}\left(t_{k}^{i} ; \Gamma_{\varepsilon, k}^{i}, p\right)$ is well defined since $\left[\mathfrak{c}_{\varepsilon, k}^{p} \backslash \Gamma_{\varepsilon, k}^{i-1}\right] \cap \mathrm{S}_{\varepsilon, k}\left(t_{k}^{i}\right)=\emptyset$.

\section{Viscous evolutions}

In this section we pass to the limit in the discrete-time problems as the time step converges to zero, for fixed $\varepsilon>0$. We prove that there exists a map $\Gamma_{\varepsilon}:[0, T] \rightarrow \mathcal{S}$, continuous with respect to the Hausdorff convergence, such that the corresponding total energy is absolutely continuous and satisfies a suitable energy balance (which was not observed in [27]). Moreover, we prove a Griffith criterion for almost every time when the tips of the crack are not contained in a certain set constituted by a finite number of points, which are either limits of the singular points of the interpolations, or limit of points of the interpolations where the energy release rate is not defined.

Definition 4.1. Fixed $\varepsilon>0$, a set function $[0, T] \ni t \mapsto \Gamma_{\varepsilon}(t) \in \mathcal{S}$ is a viscous solution in $\mathcal{S}$ if there exist time discretizations $\left\{t_{k}^{i}\right\}_{i=0}^{k}$ as in (3.1) and a sequence of set functions $[0, T] \ni t \mapsto \Gamma_{\varepsilon, k}(t) \in \mathcal{S}$ such that $\Gamma_{\varepsilon, k}(0)=\Gamma_{0}, \Gamma_{\varepsilon, k}$ is constant in every time interval $\left[t_{k}^{i}, t_{k}^{i+1}\right), \Gamma_{\varepsilon, k}\left(t_{k}^{i}\right)=\Gamma_{\varepsilon, k}^{i}$ solves (3.3) for $i \geq 1$, and

for every $t \in[0, T]$.

$$
\Gamma_{\varepsilon, k}(t) \stackrel{\mathcal{H}}{\longrightarrow} \Gamma_{\varepsilon}(t) \quad \text { as } k \rightarrow \infty
$$

Remark 4.2. Let $[0, T] \ni t \mapsto \Gamma_{\varepsilon}(t) \in \mathcal{S}$ be a viscous solution in $\mathcal{S}$. Then, by [10, Theorem 5.1], for every $t \in[0, T]$

$$
\nabla u_{\varepsilon_{k}}(t) \rightarrow \nabla u_{\varepsilon}(t) \quad \text { in } L^{2}\left(\Omega ; \mathbb{R}^{2}\right),
$$

where $u_{\varepsilon}(t):=u\left(t ; \Gamma_{\varepsilon}(t)\right)$. Moreover, since, for every $\Gamma \in \mathcal{S}, \Omega \backslash \Gamma$ is the union of a uniformly bounded number of Lipschitz open sets that intersect $\partial_{D} \Omega$, we have that for every $t \in[0, T]$

$$
u_{\varepsilon_{k}}(t) \rightarrow u_{\varepsilon}(t) \quad \text { in } H^{1}(\Omega) .
$$

We recall from [27, Proposition 5.1 and Corollary 5.2] the existence of viscous solutions and their continuity in time. We give a sketch of the proof, for the reader's convenience.

Theorem 4.3. Fixed $\varepsilon>0$, there exists a viscous solution $[0, T] \ni t \mapsto \Gamma_{\varepsilon}(t)$. Moreover, such a solution is continuous with respect to the Hausdorff convergence, its length $l_{\varepsilon}(t):=\mathcal{H}^{1}\left(\Gamma_{\varepsilon}(t)\right)$ belongs to $H^{1}(0, T)$, and there exists a positive constant $C$, independent of $t$, such that

$$
\left\|u_{\varepsilon}(t)\right\|_{H^{1}\left(\Omega \backslash \Gamma_{\varepsilon}(t)\right)} \leq C .
$$

Proof. The existence follows from the Helly Theorem, applied to the sequence of nondecreasing set functions $\Gamma_{\varepsilon, k}$. Since, for $\varepsilon$ fixed, $\left\|l_{\varepsilon, k}\right\|_{H^{1}}$ are uniformly bounded by (3.4), we have that

$$
l_{\varepsilon, k} \rightarrow l_{\varepsilon} \quad \text { in } H^{1}(0, T) .
$$

On the other hand, by (i) in Proposition 2.7 we get $\underline{l}_{\varepsilon, k} \rightarrow \underline{l}_{\varepsilon}$ pointwise, and for $t \in\left[t_{k}^{i}, t_{k}^{i+1}\right.$ )

$$
0 \leq l_{\varepsilon, k}(t)-\underline{l}_{\varepsilon, k}(t)=\int_{t_{k}^{i}}^{t} i_{\varepsilon, k}(s) \mathrm{d} s \leq \tau^{\frac{1}{2}}\left\|l_{\varepsilon, k}\right\|_{H^{1}}^{\frac{1}{2}} \leq \frac{\bar{C}}{\varepsilon} \tau^{\frac{1}{2}},
$$

where $\bar{C}$ is the constant in (3.4). Then $\underline{l}_{\varepsilon}=l_{\varepsilon}$.

The continuity of $t \mapsto \Gamma_{\varepsilon}(t)$ follows from the continuity of $l_{\varepsilon}$ (see [27, Corollary 5.2]). The functions $u_{\varepsilon_{k}}(t)$ are bounded in $H^{1}\left(\Omega \backslash \Gamma_{\varepsilon_{k}}(t)\right)$, uniformly in $k$ and $t$, by (3.4) and the regularity of the boundary datum $w$. Thus, in the limit we get (4.1).

In the following we prove some properties of the viscous solutions. Let us set

$$
\mathrm{T}_{\varepsilon}(t):=\mathrm{T}_{\Gamma_{\varepsilon}(t)}, \quad \mathrm{S}_{\varepsilon}(t):=\mathrm{S}_{\Gamma_{\varepsilon}(t)}, \quad \mathrm{R}_{\varepsilon}(t):=\mathrm{R}_{\Gamma_{\varepsilon}(t)}, \quad \mathrm{G}_{\varepsilon}(t):=\mathrm{G}_{\Gamma_{\varepsilon}(t)} .
$$

Up to considering a subsequence (depending on $\varepsilon$ ), we may assume that the number of singular points of $\Gamma_{\varepsilon, k}(T)$ is constant, so that

$$
\mathrm{S}_{\varepsilon, k}(T)=\left\{x_{\varepsilon, k}^{1}, \ldots, x_{\varepsilon, k}^{M}\right\},
$$

and $x_{\varepsilon, k}^{j} \rightarrow x_{\varepsilon}^{j}$, as $k \rightarrow \infty$. Let us define the set of limit of singular points

$$
\mathrm{F}_{\varepsilon}:=\left\{x_{\varepsilon}^{j}: x_{\varepsilon}^{j}=\lim _{k} x_{\varepsilon, k}^{j}\right\} .
$$


Notice that $\mathrm{S}_{\varepsilon}(T) \subset \mathrm{F}_{\varepsilon}$, since the curvature of every branch of any curve in $\mathcal{S}$ is less than $\frac{1}{\eta}$, and

$$
\operatorname{card}\left(\mathrm{F}_{\varepsilon}\right) \leq M=\operatorname{card}\left(\mathrm{S}_{\varepsilon, k}(T)\right) .
$$

(In fact, it might happen that $x_{\varepsilon}^{l}=x_{\varepsilon}^{j}$ for some $j$, so the inequality may be strict.) Fix now $j, l \in\{1, \ldots, M\}, j \neq l$ : since by $(2.5)\left|x_{\varepsilon, k}^{j}-x_{\varepsilon, k}^{l}\right| \geq \beta\left(\frac{2}{\tan \theta}+1\right)$ for every $k$, we have that $\left|x_{\varepsilon}^{j}-x_{\varepsilon}^{l}\right| \geq \beta\left(\frac{2}{\tan \theta}+1\right)$ for every $\varepsilon>0$.

Arguing as in [27, Lemma 6.1], we can find a partition of $[0, T]$

$$
0=t_{\varepsilon}^{0}<t_{\varepsilon}^{1}<\cdots<t_{\varepsilon}^{N_{\varepsilon}+1}=T
$$

such that for every $t \leq t^{\prime} \in[0, T]$

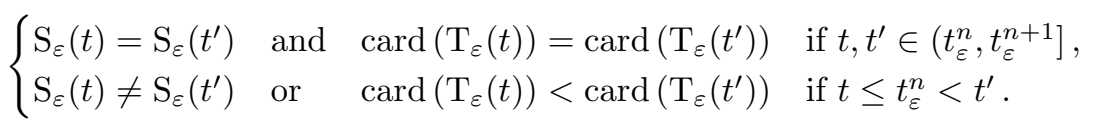

We define the time intervals

$$
I_{\varepsilon}^{n}:=\left(t_{\varepsilon}^{n}, t_{\varepsilon}^{n+1}\right] .
$$

In $I_{\varepsilon}^{n}$ we can find exactly $k_{n}=k_{n}(\varepsilon):=\operatorname{card}\left(\mathrm{T}_{\varepsilon}\left(t_{\varepsilon}^{n+1}\right)\right)$ branches parametrized by $\gamma_{\varepsilon}^{n, j}: I_{\varepsilon}^{n} \rightarrow \Omega$ with $\gamma_{\varepsilon}^{n, j}(t) \in \mathrm{T}_{\varepsilon}(t)$, for $j=1, \ldots, k_{n}$. Notice that, if a connected component $\Gamma_{\varepsilon}(t)$ belongs to $\mathcal{R}_{\eta}$, it has two tips. To simplify the notation, we see such a curve as the union of two branches, so the number of branches in $\Gamma_{\varepsilon}(t)$ equals the total number of tips. Recall that the length of any connected component is bounded from below by $(2.2)$.

Extending by continuity $\gamma_{\varepsilon}^{n, j}$ to $\overline{I_{\varepsilon}^{n}}$ we get

$$
\gamma_{\varepsilon}^{n, j}\left(\overline{I_{\varepsilon}^{n}}\right) \in \mathcal{R}_{\eta} .
$$

Let us define

$$
\overline{I_{\varepsilon}^{n}} \ni t \mapsto l_{\varepsilon}^{n, j}(t):=\mathcal{H}^{1}\left(\gamma_{\varepsilon}^{n, j}\left(\left[t_{\varepsilon}^{n}, t\right]\right)\right) .
$$

Remark 4.4. From now on we will not specify the dependence on $n$ of $\gamma_{\varepsilon}^{n, j}$ and $l_{\varepsilon}^{n, j}$. More precisely, we set

$$
\gamma_{\varepsilon}^{j}(t):=\gamma_{\varepsilon}^{n, j}(t) \quad \text { where } t \in I_{\varepsilon}^{n} .
$$

Notice that for every $t \in[0, T]$ there exists only one index $n$ such that $t \in I_{\varepsilon}^{n}$.

Since in $I_{\varepsilon}^{n}$ there are exactly $k_{n}$ branches, for every $\left(s_{1}, s_{2}\right) \subset I_{\varepsilon}^{n}$

$$
l_{\varepsilon}\left(s_{2}\right)-l_{\varepsilon}\left(s_{1}\right)=\mathcal{H}^{1}\left(\Gamma_{\varepsilon}\left(s_{2}\right) \backslash \Gamma_{\varepsilon}\left(s_{1}\right)\right)=\sum_{j=1}^{k_{n}}\left[l_{\varepsilon}^{j}\left(s_{2}\right)-l_{\varepsilon}^{j}\left(s_{1}\right)\right],
$$

which gives $l_{\varepsilon}^{j} \in H^{1}\left(I_{\varepsilon}^{i}\right)$ for every $j \in\left\{1, \ldots, k_{n}\right\}$ and

$$
i_{\varepsilon}=\sum_{j=1}^{k_{n}} i_{\varepsilon}^{j} \quad \text { a.e. in } I_{\varepsilon}^{n} .
$$

Let us define for every $t \in[0, T]$ the set

$\mathrm{B}_{\varepsilon}(t):=\left[\mathrm{T}_{\varepsilon}(t) \backslash \mathrm{G}_{\varepsilon}(t)\right] \cup\left\{p \in \mathrm{T}_{\varepsilon}(t):\right.$ there exist $\bar{k} \in \mathbb{N}, p_{k} \rightarrow p, p_{k} \in \mathrm{T}_{\varepsilon, k}(t) \backslash \mathrm{G}_{\varepsilon, k}(t)$ for $\left.k \geq \bar{k}\right\}$.

Remark 4.5. If the approximating sequence $\left(p_{k}\right)_{k}$ in the definition above is not unique, the limit point belongs to $\mathrm{F}_{\varepsilon}$. Specifically, if there are $p_{k}, q_{k} \in \mathrm{T}_{\varepsilon, k}(t)$ with $p_{k} \neq q_{k}$ and $p_{k} \rightarrow p, q_{k} \rightarrow p$, then by Proposition $2.7 p$ is limit of elements in $\mathrm{S}_{\varepsilon, k}(t)$, so $p \in \mathrm{F}_{\varepsilon}$.

Remark 4.6. If $x \in \mathrm{B}_{\varepsilon}(t) \backslash \mathrm{F}_{\varepsilon}$, then $x \in \mathrm{T}_{\varepsilon}(s)$ for every $s \in[t, T]$, in particular $x \in \mathrm{B}_{\varepsilon}(T)$. Indeed, assume $x \in \mathrm{B}_{\varepsilon}(t)$ and $x \notin \mathrm{T}_{\varepsilon}(s)$ for some $s>t$. If $x \in \mathrm{T}_{\varepsilon}(t) \backslash \mathrm{G}_{\varepsilon}(t)$ then $x \in \mathrm{S}_{\varepsilon}(T) \subset \mathrm{F}_{\varepsilon}$, since the tip in $x$ cannot be extended smoothly (see the definition of $\mathrm{G}_{\Gamma}(2.8)$ ). If $x \in \mathrm{G}_{\varepsilon}(t)$, by Remark 4.5 we can assume that there exists only one approximating sequence $\left(p_{k}\right)_{k}$ as in (4.5); then for the same arguments as in the case $x \in \mathrm{T}_{\varepsilon}(t) \backslash \mathrm{G}_{\varepsilon}(t)$ we have $p_{k} \in \mathrm{S}_{\varepsilon, k}(T)$, thus $x \in \mathrm{F}_{\varepsilon}$. 
Let us define the set of exceptional points

$$
\mathrm{E}_{\varepsilon}:=\mathrm{F}_{\varepsilon} \cup \mathrm{B}_{\varepsilon}(T) .
$$

Notice that $\mathrm{E}_{\varepsilon}$ is a finite set. Moreover, by Remark 4.6, we have

$$
\mathrm{E}_{\varepsilon}=\mathrm{F}_{\varepsilon} \cup \bigcup_{t \in[0, T]} \mathrm{B}_{\varepsilon}(t)
$$

We now present the main theorem of this section, providing an energy-dissipation balance for viscous solutions. The proof will be given in the final part of the section, after some preliminary results.

Theorem 4.7. Let $t \mapsto \Gamma_{\varepsilon}(t) \in \mathcal{S}$ be a viscous solution as in Definition 4.1. Then the total energy

$$
[0, T] \ni t \mapsto \mathcal{F}\left(t ; \Gamma_{\varepsilon}(t)\right):=\mathcal{E}\left(t ; \Gamma_{\varepsilon}(t)\right)+\mathcal{H}^{1}\left(\Gamma_{\varepsilon}(t)\right)
$$

is absolutely continuous and for every $t \in[0, T]$

$$
\begin{aligned}
& \mathcal{E}\left(t ; \Gamma_{\varepsilon}(t)\right)+\mathcal{H}^{1}\left(\Gamma_{\varepsilon}(t)\right)+\varepsilon \sum_{h=0}^{n}\left[\int_{I_{\varepsilon}^{h}} \sum_{j=1}^{k_{h}}\left(i_{\varepsilon}^{j}(s)\right)^{2} \mathrm{~d} s\right]+\varepsilon \int_{t_{\varepsilon}^{n}}^{t} \sum_{j=1}^{k_{n}}\left(i_{\varepsilon}^{j}(s)\right)^{2} \mathrm{~d} s \\
& \quad=\mathcal{E}\left(0 ; \Gamma_{0}\right)+l_{0}+\int_{0}^{t}\left\langle\nabla u_{\varepsilon}(s), \nabla \dot{w}(s)\right\rangle \mathrm{d} s,
\end{aligned}
$$

where $n$ is such that $t \in I_{\varepsilon}^{n}$. Moreover, the following hold:

(i) for every $x \in \mathrm{E}_{\varepsilon}$ ( see (4.6) for the definition of $\mathrm{E}_{\varepsilon}$ ), there exists $J \subset[0, T]$ closed interval (which can also reduce to a singleton and contains $T$ if $x \in \mathrm{B}_{\varepsilon}(T)$ ) such that

$$
x \in \mathrm{T}_{\varepsilon}(t) \text { if and only if } t \in J \text {; }
$$

(ii) for a.e. $t \in I_{\varepsilon}^{n}$ such that $\gamma_{\varepsilon}^{j}(t) \notin \mathrm{E}_{\varepsilon}$

$$
\begin{aligned}
& i_{\varepsilon}^{j}(t) \geq 0, \\
& \mathcal{G}\left(t ; \Gamma(t), \gamma_{\varepsilon}^{j}(t)\right) \leq 1+\varepsilon i_{\varepsilon}^{j}(t), \\
& {\left[-\mathcal{G}\left(t ; \Gamma(t), \gamma_{\varepsilon}^{j}(t)\right)+1+\varepsilon i_{\varepsilon}^{j}(t)\right] i_{\varepsilon}^{j}(t)=0 .}
\end{aligned}
$$

Remark 4.8. The theorem above implies that, if $\gamma_{\varepsilon}^{j}$ does not satisfy (4.8) in an interval $J \subset I_{\varepsilon}^{n}$, then $\gamma_{\varepsilon}^{j}$ is constant in $J$ and it lies on a point of the finite set $\mathrm{E}_{\varepsilon}$.

The first step in order to establish a viscous energy balance is the following chain rule (which was not proved in [27]).

Proposition 4.9. For every $n \in\left\{0, \ldots, N_{\varepsilon}\right\}$ the elastic energy $t \mapsto \mathcal{E}\left(t ; \Gamma_{\varepsilon}(t)\right)$ belongs to $\operatorname{AC}_{\text {loc }}\left(I_{\varepsilon}^{n}\right)$ and for a.e. $t \in I_{\varepsilon}^{n}$

$$
\frac{\mathrm{d}}{\mathrm{d} t} \mathcal{E}\left(t ; \Gamma_{\varepsilon}(t)\right)=-\sum_{j=1}^{k_{n}} \mathcal{G}\left(t ; \Gamma_{\varepsilon}(t), \gamma_{\varepsilon}^{j}(t)\right) \dot{l}_{\varepsilon}^{j}(t)+\left\langle\nabla u_{\varepsilon}(t), \nabla \dot{w}(t)\right\rangle,
$$

with the convention $\mathcal{G}\left(t ; \Gamma_{\varepsilon}(t), \gamma_{\varepsilon}^{j}(t)\right) i_{\varepsilon}^{j}(t)=0$ if $\gamma_{\varepsilon}^{j}(t) \notin \mathrm{G}_{\varepsilon}(t)$. Moreover, if $w \in H^{1}\left([0, T] ; H^{1}(\Omega \backslash\right.$ $\left.\left.\Gamma_{0}\right)\right), \mathcal{E}\left(\cdot ; \Gamma_{\varepsilon}(\cdot)\right)$ is $H_{\mathrm{loc}}^{1}\left(I_{\varepsilon}^{n}\right)$.

Proof. Let us fix the interval $I_{\varepsilon}^{n}$ and let $\gamma_{\varepsilon}^{1}\left(\overline{I_{\varepsilon}^{i}}\right), \ldots, \gamma_{\varepsilon}^{k_{n}}\left(\overline{I_{\varepsilon}^{n}}\right)$ be the branches that end with a tip. In $I_{\varepsilon}^{n}$ we can rewrite the elastic energy as

$$
\mathcal{E}\left(t ; \Gamma_{\varepsilon}(t)\right)=\widehat{\mathcal{E}}\left(t ; l_{\varepsilon}^{1}(t), \ldots, l_{\varepsilon}^{k_{n}}(t)\right),
$$

being $\widehat{\mathcal{E}}\left(t ; \lambda_{1}, \ldots, \lambda_{k_{n}}\right)$ the elastic energy corresponding to a boundary datum $w(t)$ and to a curve $\Gamma\left(\lambda_{1}, \ldots, \lambda_{k_{n}}\right)=\Gamma_{\varepsilon}\left(t_{\varepsilon}^{n}\right) \cup \bigcup_{j=1}^{k_{n}} C_{j}$, where $C_{j}$ is the unique curve contained in $\gamma_{\varepsilon}^{j}\left(\overline{I_{\varepsilon}^{n}}\right)$ with $\gamma_{\varepsilon}^{j}\left(t_{\varepsilon}^{n}\right) \ni$ $C_{j}$ and length $\lambda_{j}$. In fact, notice that $\Gamma\left(l_{\varepsilon}^{1}(t), \ldots, l_{\varepsilon}^{k_{n}}(t)\right)=\Gamma_{\varepsilon}(t)$.

By the properties of $\mathcal{S}$ (see Remark 2.3), for every $s \in I_{\varepsilon}^{n}$ there exists an open neighborhood $U$ of $\gamma_{j}(s)$, depending on $s$ and $j$, such that

$$
\Gamma_{\varepsilon}(s) \cap \bar{U} \in \mathcal{R}_{\eta} .
$$


Therefore, for every tip in $G_{\varepsilon}(t)$, where the energy release rate is well defined, $\widehat{\mathcal{E}}$ has partial derivatives

$$
\frac{\partial}{\partial \lambda_{j}} \widehat{\mathcal{E}}\left(t ; l_{\varepsilon}^{1}(t), \ldots, l_{\varepsilon}^{k_{n}}(t)\right)=-\mathcal{G}\left(t ; \Gamma\left(l_{\varepsilon}^{1}(t), \ldots, l_{\varepsilon}^{k_{n}}(t)\right), \gamma_{\varepsilon}^{j}(t)\right)=-\mathcal{G}\left(t ; \Gamma_{\varepsilon}(t), \gamma_{\varepsilon}^{j}(t)\right) .
$$

On the other hand, if $\gamma_{\varepsilon}^{j}(t) \notin \mathrm{G}_{\varepsilon}(t)$, then the tip does not elongate, namely $\gamma_{\varepsilon}^{j}(s)=\gamma_{\varepsilon}^{j}(t)$ for $s \in\left[t, t_{\varepsilon}^{n+1}\right]$, and $i_{\varepsilon}^{j}(s)=0$ for a.e. $s \in\left(t, t_{\varepsilon}^{n+1}\right)$. Indeed, by definition of $\mathrm{G}_{\varepsilon}(t)$, there is not an extension (see Definition 2.8) of $\Gamma_{\varepsilon}(t)$ at $\gamma_{\varepsilon}^{j}(t)$; since we are in the interval $I_{\varepsilon}^{n}$ a kinking is not created at $\gamma_{\varepsilon}^{j}(t)$.

By (4.10) and Proposition 2.12, the functions $t \mapsto \mathcal{G}\left(t ; \Gamma_{\varepsilon}(t), \gamma_{\varepsilon}^{j}(t)\right)$ are bounded in $I_{\varepsilon}^{n}$. Recalling that

$$
\frac{\partial}{\partial t} \mathcal{E}\left(t ; \Gamma_{\varepsilon}(t)\right)=\left\langle\nabla u_{\varepsilon}(t), \nabla \dot{w}(t)\right\rangle,
$$

the result follows by the chain rule.

The following proposition refines the results of [27, Lemmas 5.3, 5.5, and 5.6]. We give an independent and simplified proof for the reader's convenience. In order to simplify the notation, we omit the dependence on $\varepsilon$ for the objects that depend also on $k$.

Proposition 4.10. Let $t \in I_{\varepsilon}^{n}$ and let $j \in\left\{1, \ldots, k_{n}\right\}$ be such that $\gamma_{\varepsilon}^{j}(t) \notin \mathrm{F}_{\varepsilon}$. Define

$$
r_{j}(t):=\omega\left(\mathrm{d}\left(\gamma_{\varepsilon}^{j}(t), \mathrm{F}_{\varepsilon}\right)\right) \wedge \eta \quad \text { and } \quad \bar{s}_{j}(t):=\left[t-\left(\frac{r_{j}(t) \varepsilon}{4 \bar{C}}\right)^{2}\right] \vee t_{\varepsilon}^{n},
$$

where $\omega$ is the modulus of continuity introduced in Remark 2.3, and $\bar{C}$ is the constant in (3.4). Then there exists $\bar{k} \in \mathbb{N}$ such that for every $k>\bar{k}$ and $s \in\left(\bar{s}_{j}(t), t\right]$ the following holds:

$$
\begin{aligned}
& \mathrm{T}_{k}(s) \cap \overline{B_{r_{j}(t)}\left(\gamma_{\varepsilon}^{j}(t)\right)} \text { contains one and only one element, called } p_{k}^{j}(s), \\
& \mathrm{S}_{k}(s) \cap \overline{B_{r_{j}(t)}\left(\gamma_{\varepsilon}^{j}(t)\right)}=\emptyset, \\
& \Gamma_{k}(s) \cap \overline{B_{r_{j}(t)}\left(\gamma_{\varepsilon}^{j}(t)\right)} \in \mathcal{R}_{\eta} .
\end{aligned}
$$

Proof. For simplicity, in the proof $r$ and $\bar{s}$ stand for $r_{j}(t)$ and $\bar{s}_{j}(t)$, respectively. First, let us prove (4.11) for $s=t$. By contradiction, assume that there exist $k_{h} \rightarrow \infty$ such that at least one condition in (4.11) does not hold, for $s=t$ and $k=k_{h}$.

Consider first the case where (4.11b) does not hold, namely for every $h$ there exists $q_{h} \in \mathrm{S}_{k_{h}}(t) \cap$ $\overline{B_{r}\left(\gamma_{\varepsilon}^{j}(t)\right)}$. Then there esists $q$ such that, up to a subsequence, $q_{h} \rightarrow q$, so that $q \in \mathrm{F}_{\varepsilon} \cap \overline{B_{r}\left(\gamma_{\varepsilon}^{j}(t)\right)}$, in contradiction with the definition of $r$.

If (4.11a) does not hold (for $s=t$ and $k=k_{h}$ ), we may assume that there exist two sequences $\left(p_{h}\right)_{h}$ and $\left(q_{h}\right)_{h}$ such that

$$
p_{h}, q_{h} \in \mathrm{T}_{k_{h}}(t) \cap \overline{B_{r}\left(\gamma_{\varepsilon}^{j}(t)\right)}, \quad p_{h} \rightarrow \gamma_{\varepsilon}^{j}(t), \quad q_{h} \rightarrow q \neq \gamma_{\varepsilon}^{j}(t) .
$$

Indeed, by (ii) of Proposition 2.7, $\gamma_{\varepsilon}^{j}(t) \in \mathrm{T}_{\varepsilon}(t)$ is approximated by elements $p_{h} \in \mathrm{T}_{k_{h}}(t)$. Since (4.11a) does not hold, for every $h$ there exists $q_{h} \in \mathrm{T}_{k_{h}}(t) \cap \overline{B_{r}\left(\gamma_{\varepsilon}^{j}(t)\right)}, q_{h} \neq p_{h}$. Up to a subsequence, $q_{h} \rightarrow q$. If $q=\gamma_{\varepsilon}^{j}(t)$, then $\gamma_{\varepsilon}^{j}(t) \in \mathrm{F}_{\varepsilon}$, by (iii) of Proposition 2.7. This proves (4.12) in the case (4.11a) is not satisfied.

Notice that $p_{h}$ and $q_{h}$ belong to different branches $K_{h}^{1}$ and $K_{h}^{2}$ of $\Gamma_{k_{h}}(t)$, respectively. We have that $\mathcal{H}^{1}\left(K_{h}^{1}\right) \geq C$, for a positive $C$, since otherwise $\gamma_{\varepsilon}^{j}(t)$ is approximated by singular points and then $\gamma_{\varepsilon}^{j}(t) \in \mathrm{F}_{\varepsilon}$. Thus, let us distinguish the two cases:

$$
\mathcal{H}^{1}\left(K_{h}^{2}\right) \rightarrow 0 \quad \text { or } \quad \mathcal{H}^{1}\left(K_{h}^{2}\right) \geq C .
$$

In the first case, $q \in \mathrm{F}_{\varepsilon} \cap \overline{B_{r}\left(\gamma_{\varepsilon}^{j}(t)\right)}$, in contradiction with the definition of $r$. In the second case, passing to the limit, it is easy to see that two different branches of $\Gamma_{\varepsilon}(t)$ have nonempty intersection with $\overline{B_{r}\left(\gamma_{\varepsilon}^{j}(t)\right)}$, in contradiction with the fact that

$$
r<\omega\left(\mathrm{d}\left(\gamma_{\varepsilon}^{j}(t), \mathrm{F}_{\varepsilon}\right)\right) \leq \omega\left(\mathrm{d}\left(\gamma_{\varepsilon}^{j}(t), \mathrm{S}_{\Gamma_{\varepsilon}(t)}\right) .\right.
$$

(Recall Remark 2.3.) Then (4.11a) holds. Finally assume that (4.11c) does not hold, namely $\Gamma_{k_{h}}(t) \cap \overline{B_{r}\left(\gamma_{\varepsilon}^{j}(t)\right)} \notin \mathcal{R}_{\eta}$ for every $h$. Then $\overline{B_{r}\left(\gamma_{\varepsilon}^{j}(t)\right)}$ intersects at least two different branches of $\Gamma_{k_{h}}(t)$. (Notice that we have used the hypotesis $r<\eta$, which implies that for every branch $K$ of 
$\Gamma_{k_{h}}(t), K \cap \overline{B_{r}\left(\gamma_{\varepsilon}^{j}(t)\right)}$ is a connected component of $\Gamma_{k_{h}}(t) \cap \overline{B_{r}\left(\gamma_{\varepsilon}^{j}(t)\right)}$.) Therefore we can argue as in the previous case: on the one hand, there exists a branch converging to the branch of $\gamma_{\varepsilon}^{j}(t)$; on the other hand there exists a different branch, either converging to a point $q \in \mathrm{F}_{\varepsilon} \cap \overline{B_{r}\left(\gamma_{\varepsilon}^{j}(t)\right)}$, or with length bounded from below, cf. (4.13). This concludes the proof of (4.11) for $s=t$. Notice that we have proved also that $p_{k}^{j}(t) \rightarrow \gamma_{\varepsilon}^{j}(t)$.

We are now ready to prove (4.11) for $s \in(\bar{s}, t)$. For $k$ large

$$
\mathrm{d}\left(p_{k}^{j}(t), \gamma_{\varepsilon}^{j}(t)\right)<\frac{r}{2}
$$

and then

$$
\mathcal{H}^{1}\left(\Gamma_{k}(t) \cap \overline{B_{r}\left(\gamma_{\varepsilon}^{j}(t)\right)}\right) \geq \frac{r}{2}
$$

Let us introduce

$$
s_{k}:=\min \left\{s \in\left[t_{\varepsilon}^{n}, t\right): \Gamma_{k}(s) \cap \overline{B_{r}\left(\gamma_{\varepsilon}^{j}(t)\right)} \neq \emptyset\right\} .
$$

Notice that the set in the last definition is not empty for $k$ sufficiently large. Indeed, let $h \in \mathbb{N}$ such that $t \in\left[t_{k}^{h}, t_{k}^{h+1}\right)$. If $t \in\left(t_{k}^{h}, t_{k}^{h+1}\right)$, then $t_{k}^{h}$ is a competitor for $s_{k}$, since $\Gamma_{k}$ is piecewise constant. On the other hand, if $t=t_{k}^{h}$, then

$$
\mathcal{H}^{1}\left(\Gamma_{k}\left(t_{k}^{h}\right) \backslash \Gamma_{k}\left(t_{k}^{h-1}\right)\right) \leq \int_{t_{k}^{h-1}}^{t_{k}^{h}} \dot{l}_{k}(s) \mathrm{d} s \leq \frac{1}{\sqrt{k}}\left(\int_{t_{k}^{h-1}}^{t_{k}^{h}}\left|\dot{l}_{k}(s)\right|^{2} \mathrm{~d} s\right)^{\frac{1}{2}} \leq \frac{\bar{C}}{\varepsilon \sqrt{k}},
$$

with $\bar{C}$ the constant in (3.4); for $k$ sufficiently large this implies that $t_{k}^{h-1}$ is a competitor for $s_{k}$. Moreover, the minimum in (4.14) is attained at a node $t_{k}^{h}$, since $\Gamma_{k}$ is piecewise constant and continuous from the right, and

$$
\frac{r}{4} \leq \mathcal{H}^{1}\left(\Gamma_{k}(t) \backslash \Gamma_{k}\left(s_{k}\right)\right)
$$

for $k$ large, by (4.15).

By the monotonicity of $\Gamma_{k}$ and (4.11) for $s=t$, we obtain that for every $s \in\left(s_{k}, t\right]$

$$
\begin{aligned}
& \mathrm{T}_{k}(s) \cap \overline{B_{r}\left(\gamma_{\varepsilon}^{j}(t)\right)}=\left\{p_{k}^{j}(s)\right\}, \\
& \mathrm{S}_{k}(s) \cap \overline{B_{r}\left(\gamma_{\varepsilon}^{j}(t)\right)}=\emptyset, \\
& \Gamma_{k}(s) \cap \overline{B_{r}\left(\gamma_{\varepsilon}^{j}(t)\right)} \in \mathcal{R}_{\eta} .
\end{aligned}
$$

Therefore, the proof is completed if we show that $s_{k} \leq \bar{s}$.

Let $t \in\left(t_{k}^{h_{2}}, t_{k}^{h_{2}+1}\right)$ and $s_{k}=t_{k}^{h_{1}}$. Necessarily $h_{1}<h_{2}$, because otherwise $\Gamma_{k}(t)=\Gamma_{k}\left(s_{k}\right)$. By (3.4), we have that

$$
\frac{r}{4} \leq \mathcal{H}^{1}\left(\Gamma_{k}(t) \backslash \Gamma_{k}\left(s_{k}\right)\right)=\int_{h_{1} \tau}^{h_{2} \tau} i_{k}(s) \mathrm{d} s \leq \sqrt{\frac{\left(h_{2}-h_{1}\right)}{k}}\left(\int_{h_{1} \tau}^{h_{2} \tau}\left|i_{k}(s)\right|^{2} \mathrm{~d} s\right)^{\frac{1}{2}} \leq \frac{\bar{C}}{\varepsilon} \sqrt{t-s_{k}} .
$$

Then

$$
s_{k} \leq \bar{t}-\left(\frac{r \varepsilon}{4 \bar{C}}\right)^{2}
$$

and this concludes the proof, since $s_{k} \geq t_{\varepsilon}^{n}$.

Remark 4.11. In Proposition 4.10 we chose the notation $r_{j}(t)$ and $\bar{s}_{j}(t)$ since these quantities depend on $t$ and on the branch that we consider, which corresponds to a certain $j \in\left\{1, \ldots, k_{n}\right\}$. Moreover

$$
p_{k}^{j}(t) \rightarrow \gamma_{\varepsilon}^{j}(t)
$$

for every $t \in I_{\varepsilon}^{n}$ such that $\gamma_{\varepsilon}^{j}(t) \notin \mathrm{F}_{\varepsilon}$.

Let us fix $n \in\left\{0, \ldots, N_{\varepsilon}\right\}, j \in\left\{1, \ldots, k_{n}\right\}$, and $t \in I_{\varepsilon}^{n}$ such that $\gamma_{\varepsilon}^{j}(t) \notin \mathrm{F}_{\varepsilon}$. With the notation of Proposition 4.10, for $k$ sufficiently large and $s \in\left(\bar{s}_{j}(t), t\right]$, we have that

$$
\Gamma_{k}(s) \cap \overline{B_{r_{j}(t)}\left(\gamma_{\varepsilon}^{j}(t)\right)} \in \mathcal{R}_{\eta} .
$$


Let us consider the functions

$$
\begin{aligned}
s \in\left(\bar{s}_{j}(t), t\right] \mapsto \underline{\ell}_{k}^{j}(s):= & \mathcal{H}^{1}\left(\Gamma_{k}(s) \cap B_{r_{j}(t)}\left(\gamma_{\varepsilon}^{j}(t)\right)\right), \\
s \in\left(\bar{s}_{j}(t), t\right] \mapsto \ell_{k}^{j}(s):= & \mathcal{H}^{1}\left(\Gamma_{k}(s) \cap B_{r_{j}(t)}\left(\gamma_{\varepsilon}^{j}(t)\right)\right) \\
& \quad+\frac{s-\underline{\tau}_{k}(s)}{\tau} \mathcal{H}^{1}\left(\left[\Gamma_{k}(s+\tau) \backslash \Gamma_{k}(s)\right] \cap B_{r_{j}(t)}\left(\gamma_{\varepsilon}^{j}(t)\right)\right), \\
s \in\left(\bar{s}_{j}(t), t\right] \mapsto \ell_{\varepsilon}^{j}(s):= & \mathcal{H}^{1}\left(\Gamma(s) \cap B_{r_{j}(t)}\left(\gamma_{\varepsilon}^{j}(t)\right)\right)=l_{\varepsilon}^{j}(s)-\mathcal{H}^{1}\left(\gamma_{\varepsilon}^{j}\left(\left[t_{\varepsilon}^{i}, t\right]\right) \backslash B_{r_{j}(t)}\left(\gamma_{\varepsilon}^{j}(t)\right)\right),
\end{aligned}
$$

where $\underline{\tau}_{k}(s):=t_{k}^{h}$ if $s \in\left[t_{k}^{h}, t_{k}^{h+1}\right)$. Since $\Gamma_{k}(s) \stackrel{\mathcal{H}}{\longrightarrow} \Gamma(s)$ for every $s \in[0, T]$, we get that

$$
\underline{\ell}_{k}^{j}(s) \rightarrow \ell_{\varepsilon}^{j}(s) \text { for every } s \in\left(\bar{s}_{j}(t), t\right] .
$$

Arguing as in the proof of Theorem 4.3 we have that

$$
\left\|\ell_{k}^{j}\right\|_{H^{1}\left(\bar{s}_{j}(t), t\right)} \leq C,
$$

with $C$ depending only on the data of the problem and on $\varepsilon$, and that

$$
\ell_{k}^{j}(s) \rightarrow \ell_{\varepsilon}^{j}(s) \quad \text { in }\left(\bar{s}_{j}(t), t\right], \quad \ell_{k}^{j} \rightarrow \ell_{\varepsilon}^{j} \quad \text { in } H^{1}\left(\bar{s}_{j}(t), t\right) .
$$

Notice that

$$
\dot{\ell}_{k}^{j}(s)=v_{k}\left(s, p_{k}^{j}(s)\right), \quad \dot{\ell}_{\varepsilon}^{j}(s)=\dot{l}_{\varepsilon}^{j}(s) .
$$

We employ the following result, proved in [27, Lemma 7.3], which holds since $\bar{s}_{j}(t)<t$.

Lemma 4.12. Let us consider $\left(s_{1}, s_{2}\right) \subset I_{\varepsilon}^{n}$ such that $\gamma_{\varepsilon}^{j}(\tilde{t}) \notin \mathrm{F}_{\varepsilon}$ for every $\tilde{t} \in\left(s_{1}, s_{2}\right)$. Then, for every $\tilde{t} \in\left(s_{1}, s_{2}\right)$ there exists a set $A_{j}(\tilde{t}) \subset\left(s_{1}, s_{2}\right)$, at most countable, such that $\left(\bar{s}_{j}\left(t_{1}\right), t_{1}\right]$ and $\left(\bar{s}_{j}\left(t_{2}\right), t_{2}\right]$ are disjoint for $t_{1} \neq t_{2} \in A_{j}(\tilde{t})$ and

$$
\left(s_{1}, \tilde{t}\right]=\bigcup_{t \in A_{j}(\tilde{t})}\left(\bar{s}_{j}(t), t\right] .
$$

Employing the above lemma, we deduce the following convergence result.

Lemma 4.13. For every $\left(\bar{t}_{1}, \bar{t}_{2}\right) \subset \subset\left(s_{1}, s_{2}\right)$

$$
v_{k}\left(\cdot, p_{k}^{j}(\cdot)\right) \rightarrow i_{\varepsilon}^{j} \quad \text { in } L^{2}\left(\bar{t}_{1}, \bar{t}_{2}\right) .
$$

Proof. Let $f \in L^{2}\left(\bar{t}_{1}, \bar{t}_{2}\right)$. We have that

$$
\int_{\bar{t}_{1}}^{\bar{t}_{2}} v_{k}\left(s, p_{k}^{j}(s)\right) f(s) \mathrm{d} s=\sum_{t \in A_{j}\left(\bar{t}_{1}\right)_{\left(\bar{s}_{j}(t), t\right] \cap\left(\bar{t}_{1}, \bar{t}_{2}\right)}} v_{k}\left(s, p_{k}^{j}(s)\right) f(s) \mathrm{d} s,
$$

and, by (4.17),

$$
v_{k}\left(\cdot, p_{k}^{j}(\cdot)\right) \rightarrow i_{\varepsilon}^{j} \quad \text { in } L^{2}\left(\bar{s}_{j}(t), t\right)
$$

for every $t \in A_{j}\left(\bar{t}_{1}\right)$. Lemma 4.12 ensures that $A_{j}\left(\bar{t}_{1}\right)$ is at most countable, so the countable additivity of the integral allows us to obtain (4.18).

We are now in the position to prove a Griffith criterion for viscous solutions.

Proposition 4.14. Let $n \in\left\{0, \ldots, N_{\varepsilon}\right\}, j \in\left\{1, \ldots, k_{n}\right\}$, and $\left(s_{1}, s_{2}\right) \subset I_{\varepsilon}^{n}$ such that $\gamma_{\varepsilon}^{j}(\tilde{t}) \notin \mathrm{E}_{\varepsilon}$ for every $\tilde{t} \in\left(s_{1}, s_{2}\right)$. Then

$$
\left(s_{1}, s_{2}\right) \ni t \mapsto \mathcal{G}\left(t ; \Gamma(t), \gamma_{\varepsilon}^{j}(t)\right) \quad \text { is continuous }
$$

and for a.e. $t \in\left(s_{1}, s_{2}\right)$ the following conditions hold:

$$
\begin{aligned}
& i_{\varepsilon}^{j}(t) \geq 0, \\
& \mathcal{G}\left(t ; \Gamma(t), \gamma_{\varepsilon}^{j}(t)\right) \leq 1+\varepsilon i_{\varepsilon}^{j}(t), \\
& {\left[-\mathcal{G}\left(t ; \Gamma(t), \gamma_{\varepsilon}^{j}(t)\right)+1+\varepsilon l_{\varepsilon}^{j}(t)\right] l_{\varepsilon}^{j}(t)=0 .}
\end{aligned}
$$


Proof. For every $\Gamma \in \mathcal{S}$ and $p \in \mathrm{G}_{\Gamma}$, we denote

$$
\mathcal{G}_{k}(t ; \Gamma, p):=\mathcal{G}\left(w_{k}(t) ; \Gamma, p\right) .
$$

Recalling the definition of $\mathrm{E}_{\varepsilon}$ (4.6), we employ Proposition 2.12 and (4.16) to deduce (4.19) and the convergence

$$
\mathcal{G}_{k}\left(t ; \Gamma_{k}(t), p_{k}^{j}(t)\right) \rightarrow \mathcal{G}\left(t ; \Gamma(t), \gamma_{\varepsilon}^{j}(t)\right) \quad \text { for every } t \in\left(s_{1}, s_{2}\right) .
$$

By (3.5), we have that for every $t \in\left(s_{1}, s_{2}\right)$

$$
\begin{aligned}
& v_{k}\left(t, p_{k}^{j}(t)\right) \geq 0, \\
& \mathcal{G}_{k}\left(t ; \Gamma_{k}(t), p_{k}^{j}(t)\right) \leq 1+\varepsilon v_{k}\left(t, p_{k}^{j}(t)\right), \\
& {\left[-\mathcal{G}_{k}\left(t ; \Gamma_{k}(t), p_{k}^{j}(t)\right)+1+\varepsilon v_{k}\left(t, p_{k}^{j}(t)\right)\right] v_{k}\left(t, p_{k}^{j}(t)\right)=0 .}
\end{aligned}
$$

Since $\mathcal{G}_{k}\left(t ; \Gamma_{k}(t), p_{k}^{j}(t)\right) \geq 0$, by (4.18) and (4.22b) the functions

$$
\left(\bar{t}_{1}, \bar{t}_{2}\right) \ni s \mapsto \mathcal{G}_{k}\left(s ; \Gamma_{k}(s), p_{k}^{j}(s)\right)
$$

are equibounded in $L^{2}\left(\bar{t}_{1}, \bar{t}_{2}\right)$, for every $\left(\bar{t}_{1}, \bar{t}_{2}\right) \subset \subset\left(s_{1}, s_{2}\right)$. By the pointwise convergence $(4.21)$ we get that

$$
\mathcal{G}_{k}\left(\cdot ; \Gamma_{k}(\cdot), p_{k}^{j}(\cdot)\right) \rightarrow \mathcal{G}\left(\cdot ; \Gamma(\cdot), \gamma_{\varepsilon}^{j}(\cdot)\right) \quad \text { in } L^{2}\left(\bar{t}_{1}, \bar{t}_{2}\right) .
$$

Integrating (4.22b) in every $\left(\bar{t}_{1}, \bar{t}_{2}\right)$, and passing to the limit using (4.18) and (4.26), we obtain that

$$
\int_{\bar{t}_{1}}^{\bar{t}_{2}} \mathcal{G}\left(s ; \Gamma(s), \gamma_{\varepsilon}^{j}(s)\right) \mathrm{d} s \leq \int_{\bar{t}_{1}}^{\bar{t}_{2}} 1+\varepsilon i_{\varepsilon}^{j}(s) \mathrm{d} s .
$$

Therefore we deduce inequality (4.20b) in the Lebesgue points of $l_{\varepsilon}^{j}$ in $\left(s_{1}, s_{2}\right)$.

Again by (4.23),

$$
\int_{\bar{t}_{1}}^{\bar{t}_{2}} \mathcal{G}_{k}\left(s ; \Gamma_{k}(s), p_{k}^{j}(s)\right) \mathrm{d} s \rightarrow \int_{\bar{t}_{1}}^{\bar{t}_{2}} \mathcal{G}\left(s ; \Gamma(s), \gamma_{\varepsilon}^{j}(s)\right) \mathrm{d} s,
$$

and, since $\mathcal{G}_{k}\left(t ; \Gamma_{k}(t), p_{k}^{j}(t)\right) \geq 0$, we get

$$
\mathcal{G}_{k}\left(\cdot ; \Gamma_{k}(\cdot), p_{k}^{j}(\cdot)\right) \rightarrow \mathcal{G}\left(\cdot ; \Gamma(\cdot), \gamma_{\varepsilon}^{j}(\cdot)\right) \quad \text { in } L^{1}\left(\bar{t}_{1}, \bar{t}_{2}\right) .
$$

Moreover, the continous function $s \mapsto \mathrm{d}\left(\gamma_{\varepsilon}^{j}(s), \mathrm{F}_{\varepsilon}\right)$ has positive minimum in $\left[\bar{t}_{1}, \bar{t}_{2}\right]$. Then there exists a positive constant $C_{0}$ such that, using the notation of Proposition 4.10,

$$
r_{j}(s) \geq C_{0} \quad \text { for every } s \in\left[\bar{t}_{1}, \bar{t}_{2}\right] .
$$

Let us now fix a subinterval $\left(\bar{s}_{j}(t), t\right] \subset\left(s_{1}, s_{2}\right)$. By Proposition 4.10, there exists $\bar{k}$ such that $(4.11)$ holds for $k \geq \bar{k}$ and $s \in\left(\bar{s}_{j}(t), t\right]$. Thanks to (4.11) and to the fact that (2.12) holds with a radius $r=C_{0}$ independent of $s$ by (4.25), we are allowed to use Proposition 2.12. Therefore, by $(2.13)$ and (4.1), we get that there exists a positive constant $C$ independent of $k \geq \bar{k}$ and $s \in\left(\bar{s}_{j}(t), t\right]$ such that

Using also (4.24), it follows that

$$
\mathcal{G}_{k}\left(s ; \Gamma_{k}(s), p_{k}^{j}(s)\right) \leq C \quad \text { for every } k \geq \bar{k} \text { and } s \in\left(\bar{s}_{j}(t), t\right] .
$$

$$
\mathcal{G}_{k}\left(\cdot ; \Gamma_{k}(\cdot), p_{k}^{j}(\cdot)\right) \rightarrow \mathcal{G}\left(\cdot ; \Gamma(\cdot), \gamma_{\varepsilon}^{j}(\cdot)\right) \quad \text { in } L^{q}\left(\bar{s}_{j}(t), t\right), \quad \text { for every } q \in[1,+\infty) .
$$

Let us now prove $(4.20 \mathrm{c})$. It follows immediately from (4.20a) and (4.20b) that

$$
\left[-\mathcal{G}\left(t ; \Gamma(t), \gamma_{\varepsilon}^{j}(t)\right)+1+\varepsilon i_{\varepsilon}^{j}(t)\right] i_{\varepsilon}^{j}(t) \geq 0 .
$$

By (4.18), (4.22c), and (4.24) we deduce that

$$
\begin{aligned}
0 & \leq \int_{\bar{s}_{j}(t)}^{t}\left[-\mathcal{G}\left(s ; \Gamma(s), \gamma_{\varepsilon}^{j}(s)\right)+1+\varepsilon i_{\varepsilon}^{j}(s)\right] i_{\varepsilon}^{j}(s) \mathrm{d} s \\
& \leq \lim _{k \rightarrow \infty} \int_{\bar{s}_{j}(t)}^{t}\left[-\mathcal{G}_{k}\left(s ; \Gamma_{k}(s), p_{k}^{j}(s)\right)+1\right] v_{k}\left(s, p_{k}^{j}(s)\right) \mathrm{d} s+\varepsilon \liminf _{k \rightarrow \infty} \int_{\bar{s}_{j}(t)}^{t} v_{k}\left(s, p_{k}^{j}(s)\right)^{2} \mathrm{~d} s \\
& \leq \liminf _{k \rightarrow \infty} \int_{\bar{s}_{j}(t)}^{t}\left[-\mathcal{G}_{k}\left(s ; \Gamma_{k}(s), p_{k}^{j}(s)\right)+1+\varepsilon v_{k}\left(s, p_{k}^{j}(s)\right)\right] v_{k}\left(s, p_{k}^{j}(s)\right) \mathrm{d} s=0 .
\end{aligned}
$$

Then (4.20c) holds in the Lebesgue points of $i_{\varepsilon}^{j}$ in $\left(s_{1}, s_{2}\right)$, and the proof is completed. 
We are now ready to prove Theorem 4.7.

Proof of Theorem 4.7. Let us fix $n \in\left\{0, \ldots, N_{\varepsilon}\right\}$ and $j \in\left\{1, \ldots, k_{n}\right\}$, and let us consider the intersections of $\gamma_{\varepsilon}^{j}$ with the set $\mathrm{F}_{\varepsilon}$ defined in (4.2): since $\Gamma_{\varepsilon}$ is nondecreasing and the curves of $\mathcal{S}$ have no self-intersections, if $\bar{x} \in \mathrm{F}_{\varepsilon} \cap \gamma_{\varepsilon}^{j}\left(I_{\varepsilon}^{n}\right)$, then there are $t_{\varepsilon}^{n} \leq \bar{t}_{1} \leq \bar{t}_{2} \leq t_{\varepsilon}^{n+1}$ such that

$$
\gamma_{\varepsilon}^{j}(s)=\bar{x} \quad \text { if and only if } s \in\left[\bar{t}_{1}, \bar{t}_{2}\right]
$$

By Remark 4.6, if $x \in \mathrm{B}_{\varepsilon}(T)$, we have that the tip stops in $x$ until the final time $T$, and we deduce in particular (4.27) for $\bar{t}_{2}=t_{\varepsilon}^{n+1}$.

Therefore (i) holds and $\left(t_{\varepsilon}^{n}, t_{\varepsilon}^{n+1}\right)$ is the union of a finite number of open intervals where $\gamma_{\varepsilon}^{j}(t) \notin$ $\mathrm{E}_{\varepsilon}$, and of a finite number of closed intervals in each of which $\gamma_{\varepsilon}^{j}(t)$ is constant and belongs to $\mathrm{E}_{\varepsilon}$. Combining this observation with Proposition 4.14 gives the Griffith conditions (4.8).

When $\bar{t}_{1}<\bar{t}_{2}$ in $(4.27)$, we can say that

$$
i_{\varepsilon}^{j}(s)=0 \quad \text { for } s \in\left(\bar{t}_{1}, \bar{t}_{2}\right) .
$$

By (4.20c), we have that for every $n, j$, and for a.e. $t \in I_{\varepsilon}^{n}$

$$
\left[-\mathcal{G}\left(t ; \Gamma(t), \gamma_{\varepsilon}^{j}(t)\right)+1+\varepsilon i_{\varepsilon}^{j}(t)\right] i_{\varepsilon}^{j}(t)=0 .
$$

Therefore, recalling (4.4) and $(4.9), \mathcal{F}\left(\cdot ; \Gamma_{\varepsilon}(\cdot)\right)$ defined in $(2.1)$ is absolutely continuous in every $\left(s_{1}, s_{2}\right) \subset I_{\varepsilon}^{n}$ and

$$
\begin{aligned}
\frac{\mathrm{d}}{\mathrm{d} t} \mathcal{F}\left(t ; \Gamma_{\varepsilon}(t)\right) & =\sum_{j=1}^{k_{n}}\left[-\mathcal{G}\left(t ; \Gamma_{\varepsilon}(t), \gamma_{\varepsilon}^{j}(t)\right)+1\right] i_{\varepsilon}^{j}(t)+\left\langle\nabla u_{\varepsilon}(t), \nabla \dot{w}(t)\right\rangle \\
& =-\varepsilon \sum_{j=1}^{k_{n}}\left(i_{\varepsilon}^{j}(t)\right)^{2}+\left\langle\nabla u_{\varepsilon}(t), \nabla \dot{w}(t)\right\rangle .
\end{aligned}
$$

with the convention $\mathcal{G}\left(t ; \Gamma_{\varepsilon}(t), \gamma_{\varepsilon}^{j}(t)\right) i_{\varepsilon}^{j}(t)=0$ if $\gamma_{\varepsilon}^{j}(t) \notin \mathrm{G}_{\varepsilon}(t)$. Integrating,

$$
\mathcal{F}\left(s_{2} ; \Gamma_{\varepsilon}\left(s_{2}\right)\right)-\mathcal{F}\left(s_{1} ; \Gamma_{\varepsilon}\left(s_{1}\right)\right)=\int_{s_{1}}^{s_{2}}\left[-\varepsilon \sum_{j=1}^{k_{n}}\left(j_{\varepsilon}^{j}(s)\right)^{2}+\left\langle\nabla u_{\varepsilon}(s), \nabla \dot{w}(s)\right\rangle\right] \mathrm{d} s .
$$

We can now pass to the limit as $s_{1} \rightarrow t_{\varepsilon}^{n}$ and $s_{2} \rightarrow t_{\varepsilon}^{n+1}$, in view of the continuity of $\mathcal{F}\left(\cdot ; \Gamma_{\varepsilon}(\cdot)\right)$ and of the fact that $\left[-\varepsilon \sum_{j=1}^{k_{n}}\left(i_{\varepsilon}^{j}(t)\right)^{2}+\left\langle\nabla u_{\varepsilon}(t), \nabla \dot{w}(t)\right\rangle\right] \in L^{1}\left(I_{\varepsilon}^{n}\right)$, obtaining the energy balance in every $I_{\varepsilon}^{n}$. Since the number of such intervals is finite and $\mathcal{F}\left(\cdot ; \Gamma_{\varepsilon}(\cdot)\right)$ is continuous in $[0, T]$, summing up we obtain (4.7). This concludes the proof.

\section{The VAnishing Viscosity Limit}

In this section we pass to the limit in the viscous solutions as the viscosity parameter $\varepsilon$ tends to zero. The limit evolution may display jumps in time. In order to provide a better description of the system during jumps we reparametrize by arc-length the viscous solutions, in such a way that we get a family of Lipschitz evolutions. In the limit, we obtain an evolution parametrized by arc-length, where jumps are described by means of a slow time scale. This technique was already employed in $[11,15,7,19,20]$.

Given a family of viscous solutions $\left(\Gamma_{\varepsilon}\right)_{\varepsilon>0}$ as in Definition 4.1, for $t \in[0, T]$ we set

$$
s_{\varepsilon}^{\circ}(t):=t+\mathcal{H}^{1}\left(\Gamma_{\varepsilon}(t) \backslash \Gamma_{0}\right)=t+\left(l_{\varepsilon}(t)-l_{0}\right) .
$$

Being $l_{\varepsilon}$ increasing, we get that $s_{\varepsilon}^{\circ}$ is strictly increasing and absolutely continuous and that

$$
s_{\varepsilon}^{\circ}\left(t_{2}\right)-s_{\varepsilon}^{\circ}\left(t_{1}\right) \geq t_{2}-t_{1} \quad \text { for every } 0 \leq t_{1} \leq t_{2} \leq S_{\varepsilon}:=s_{\varepsilon}^{\circ}(T) .
$$

Let $t_{\varepsilon}^{\circ}:\left[0, S_{\varepsilon}\right] \mapsto[0, T]$ be the inverse of $s_{\varepsilon}^{\circ}$; then $t_{\varepsilon}^{\circ}$ is strictly increasing. By the uniform bound on the length of the elements of $\mathcal{S}$, it follows that $\bar{S}:=\sup _{\varepsilon} S_{\varepsilon}<+\infty$ and then, for a sequence $\varepsilon_{k}$, $S_{\varepsilon_{k}} \rightarrow S$, with $S \geq T$. By setting $t_{\varepsilon}^{\circ}(t)=t_{\varepsilon}^{\circ}\left(S_{\varepsilon}\right)$ on $\left(S_{\varepsilon}, \bar{S}\right]$, we may assume that $t_{\varepsilon}^{\circ}$ is defined on the fixed time interval $[0, S]$. For $s \in[0, S]$ we set

$$
l_{\varepsilon}^{\circ}(s):=l_{\varepsilon}\left(t_{\varepsilon}^{\circ}(s)\right), \quad \Gamma_{\varepsilon}^{\circ}(s):=\Gamma_{\varepsilon}\left(t_{\varepsilon}^{\circ}(s)\right), \quad u_{\varepsilon}^{\circ}(s):=u_{\varepsilon}\left(t_{\varepsilon}^{\circ}(s)\right) .
$$


Definition 5.1. A rescaled approximable quasistatic evolution is a function $s \mapsto\left(\Gamma^{\circ}(s), t^{\circ}(s)\right)$, defined in $[0, S]$, with values in $\mathcal{S} \times[0, T]$, such that there is a sequence $\Gamma_{\varepsilon_{k}}$ of viscous solutions in $\mathcal{S}$, with $\varepsilon_{k} \rightarrow 0$, for which the following hold:

$$
\begin{array}{ll}
\Gamma_{\varepsilon_{k}}^{\circ}(s) \stackrel{\mathcal{H}}{\longrightarrow} \Gamma^{\circ}(s) & \text { for every } s \in[0, S], \\
t_{\varepsilon_{k}}^{\circ} \stackrel{*}{\rightarrow} t^{\circ} & \text { in } W^{1, \infty}([0, S]),
\end{array}
$$

where $\Gamma_{\varepsilon}^{\circ}$ and $t_{\varepsilon}^{\circ}$ are as above, see (5.1)-(5.2).

Employing the Helly Theorem for families of nondecreasing set functions, in the following proposition we prove the existence of rescaled approximable quasistatic evolutions.

Proposition 5.2. There exists a rescaled approximable quasistatic evolution. Moreover, for every rescaled approximable quasistatic evolution $s \mapsto\left(\Gamma^{\circ}(s), t^{\circ}(s)\right)$ the following hold (with the notation as above): the set function $s \mapsto \Gamma^{\circ}(s)$ is nondecreasing,

$$
l_{\varepsilon_{k}}^{\circ} \stackrel{*}{\rightarrow} l^{\circ} \quad \text { in } W^{1, \infty}([0, S]),
$$

and

$$
\left(t^{\circ}\right)^{\prime}(s)+\left(l^{\circ}\right)^{\prime}(s)=1 \quad \text { for a.e. } s \in(0, S),
$$

where $l^{\circ}(s):=\mathcal{H}^{1}\left(\Gamma^{\circ}(s)\right)$ for every $s \in[0, S]$ and the symbol' denotes the derivative with respect to $s$. Furthermore, setting $u^{\circ}(s):=u\left(t^{\circ}(s), \Gamma^{\circ}(s)\right)$, we have that for every $s$

$$
\nabla u_{\varepsilon_{k}}^{\circ}(s) \rightarrow \nabla u^{\circ}(s) \quad \text { in } L^{2}\left(\Omega ; \mathbb{R}^{2}\right) .
$$

Proof. By (5.1) we get $s=t_{\varepsilon}^{\circ}(s)+\left(l_{\varepsilon}^{\circ}(s)-l_{0}\right)$, and taking the derivative we obtain the identity

$$
\left(t_{\varepsilon}^{\circ}\right)^{\prime}(s)+\left(l_{\varepsilon}^{\circ}\right)^{\prime}(s)=1 \text { for every } \varepsilon \text { and } s .
$$

Therefore $t_{\varepsilon}^{\circ}$ and $l_{\varepsilon}^{\circ}$ are families of contractions on $[0, S]$. There are a subsequence $\varepsilon_{k}$ and functions $t^{\circ}, l \in W^{1, \infty}([0, S])$ such that

$$
t_{\varepsilon_{k}}^{\circ} \stackrel{*}{\rightarrow} t^{\circ}, \quad l_{\varepsilon_{k}}^{\circ} \stackrel{*}{\rightarrow} l \quad \text { in } W^{1, \infty}([0, S]) .
$$

Moreover, the Helly Theorem applies to the family of nondecreasing set functions $s \mapsto \Gamma_{\varepsilon}^{\circ}(s)$, so there exists $s \mapsto \Gamma^{\circ}(s) \in \mathcal{S}$ nondecreasing and a further subsequence of $\varepsilon_{k}$ (not relabeled) such that

$$
\Gamma_{\varepsilon_{k}}^{\circ}(s) \stackrel{\mathcal{H}}{\longrightarrow} \Gamma^{\circ}(s) \text { for every } s \in[0, S],
$$

namely (5.3a) holds. By the properties of $\mathcal{S}$, this implies that $\mathcal{H}^{1}\left(\Gamma_{\varepsilon_{k}}^{\circ}(s)\right) \rightarrow \mathcal{H}^{1}\left(\Gamma^{\circ}(s)\right)$ for every $s$. Recalling (5.7), we get $l=l^{\circ}$ and (5.4). Finally, (5.5) follows by (5.3a) and [10, Theorem 5.1]. This concludes the proof.

In the following part of this section, we derive important properties of rescaled approximable quasistatic evolutions. We define

$$
\begin{array}{ll}
s_{-}^{\circ}(t):=\sup \left\{s \in[0, S]: t^{\circ}(s)<t\right\} & \text { for } t \in(0, T], \\
s_{+}^{\circ}(t):=\inf \left\{s \in[0, S]: t^{\circ}(s)>t\right\} & \text { for } t \in[0, T),
\end{array}
$$

and $s_{-}^{\circ}(0):=0, s_{+}^{\circ}(T):=S$. By standard arguments, we have that

$$
\begin{gathered}
s_{-}^{\circ}(t) \leq \liminf _{\varepsilon \rightarrow 0} s_{\varepsilon}^{\circ}(t) \leq \limsup _{\varepsilon \rightarrow 0} s_{\varepsilon}^{\circ}(t) \leq s_{+}^{\circ}(t) \quad \text { and } \quad t^{\circ}\left(s_{-}^{\circ}(t)\right)=t=t^{\circ}\left(s_{+}^{\circ}(t)\right), \quad \text { for } t \in[0, T], \\
s_{-}^{\circ}\left(t^{\circ}(s)\right) \leq s \leq s_{+}^{\circ}\left(t^{\circ}(s)\right) \quad \text { for } s \in[0, S], \\
S^{\circ}:=\left\{t \in[0, T]: s_{-}^{\circ}(t)<s_{+}^{\circ}(t)\right\} \quad \text { is at most countable, } \\
U^{\circ}:=\left\{s \in[0, S]: t^{\circ} \text { is constant in a neighborhood of } s\right\}=\bigcup_{t \in S^{\circ}}\left(s_{-}^{\circ}(t), s_{+}^{\circ}(t)\right) .
\end{gathered}
$$

As in the previous section, we now divide $[0, T]$ in subintervals where the number of branches of $\Gamma^{\circ}$ is constant. Such branches are in turn limits of branches of viscous solutions. Once these approximation properties are ready, we will adapt the arguments of [15] and [19]. Let us set

$$
\mathrm{T}^{\circ}(s):=\mathrm{T}_{\Gamma^{\circ}(s)}, \quad \mathrm{S}^{\circ}(s):=\mathrm{S}_{\Gamma^{\circ}(s)}, \quad \mathrm{R}^{\circ}(s):=\mathrm{R}_{\Gamma^{\circ}(s)}, \quad \mathrm{G}^{\circ}(s):=\mathrm{B}_{\Gamma^{\circ}(s)} .
$$

Up to extracting a further subsequence, we may assume that the sets $\mathrm{F}_{\varepsilon}$ introduced in (4.2) are such that

$$
\mathrm{F}_{\varepsilon}=\left\{x_{\varepsilon}^{1}, \ldots, x_{\varepsilon}^{M}\right\}
$$


with $M$ independent of $\varepsilon$, and $x_{\varepsilon}^{j} \rightarrow x_{j}$ as $\varepsilon \rightarrow 0$. Recall that $\mathrm{S}_{\varepsilon}(T) \subset \mathrm{F}_{\varepsilon}$. We define the set of limit points

$$
\mathrm{F}:=\left\{x^{j}: x^{j}=\lim _{\varepsilon} x_{\varepsilon}^{j}\right\}
$$

We have that $\operatorname{card}(\mathrm{F}) \leq M$ and $\left|x^{j}-x^{l}\right| \geq \beta\left(\frac{2}{\tan \theta}+1\right)$ for every $x^{j} \neq x^{l} \in \mathrm{F}$. Moreover, we can find a partition of $[0, S]$

$$
0=s^{0}<s^{1}<\cdots<s^{N+1}=S
$$

such that for every $s \leq s^{\prime} \in[0, S]$

$$
\begin{cases}\mathrm{S}^{\circ}(s)=\mathrm{S}^{\circ}\left(s^{\prime}\right) \text { and } \operatorname{card}\left(\mathrm{T}^{\circ}(s)\right)=\operatorname{card}\left(\mathrm{T}^{\circ}\left(s^{\prime}\right)\right) & \text { if } s, s^{\prime} \in\left(s^{n}, s^{n+1}\right], \\ \mathrm{S}^{\circ}(s) \neq \mathrm{S}^{\circ}\left(s^{\prime}\right) \text { or } \quad \operatorname{card}\left(\mathrm{T}^{\circ}(s)\right)<\operatorname{card}\left(\mathrm{T}^{\circ}\left(s^{\prime}\right)\right) & \text { if } s \leq s^{n}<s^{\prime} .\end{cases}
$$

As in the previous section, in the time intervals

$$
I_{n}^{\circ}:=\left(s^{n}, s^{n+1}\right]
$$

we can find exactly $h_{n}$ branches parametrized by $\gamma_{n, j}^{\circ}: I_{n}^{\circ} \rightarrow \Omega$, with $\gamma_{n, j}^{\circ}(s) \in \mathrm{T}^{\circ}(s)$, and

$$
\gamma_{n, j}^{\circ}\left(\overline{I_{n}^{\circ}}\right) \in \mathcal{R}_{\eta} .
$$

If we introduce the functions $\overline{I_{n}^{\circ}} \ni s \mapsto l_{n, j}^{\circ}(s):=\mathcal{H}^{1}\left(\gamma_{n, j}^{\circ}\left(\left[s^{n}, s\right]\right)\right)$, we have that for every $\left(s_{1}, s_{2}\right) \subset$ $I_{n}^{\circ}$

$$
\left(l^{\circ}\right)^{\prime}(s)=\sum_{j=1}^{h_{n}}\left(l_{n, j}^{\circ}\right)^{\prime}(s) \quad \text { in } I_{n}^{\circ} .
$$

Thus $l_{n, j}^{\circ} \in W^{1, \infty}\left(I_{n}^{\circ}\right)$ for every $j \in\left\{1, \ldots, h_{n}\right\}$, with $\left(l_{n, j}^{\circ}\right)^{\prime}(s) \leq 1$. In order to simplify the notation, in the following we omit the dependence on $n$ of $\gamma_{n, j}^{\circ}$ and $l_{n, j}^{\circ}$ (see Remark 4.4).

As in Section 4 we define for every $s \in[0, S]$ the set

$\mathrm{B}^{\circ}(s):=\left[\mathrm{T}^{\circ}(s) \backslash \mathrm{G}^{\circ}(s)\right] \cup\left\{p \in \mathrm{T}^{\circ}(s)\right.$ : there exist $\varepsilon_{0}>0, p_{\varepsilon} \rightarrow p, p_{\varepsilon} \in \mathrm{T}_{\varepsilon}^{\circ}(s) \backslash \mathrm{G}_{\varepsilon}^{\circ}(s)$ for $\left.\varepsilon<\varepsilon_{0}\right\}$ and the set of exceptional points

$$
\mathrm{E}^{\circ}:=\mathrm{F} \cup \mathrm{B}^{\circ}(T) .
$$

Remark 5.3. As in Remark 4.6, we can see that if $x \in \mathrm{B}^{\circ}(s) \backslash \mathrm{F}$, then $x \in \mathrm{T}^{\circ}(\tau)$ for every $\tau \in[s, S]$, namely $x \in \mathrm{B}^{\circ}(S)$. In particular,

$$
\mathrm{E}^{\circ}=\mathrm{F} \cup \bigcup_{s \in[0, S]} \mathrm{B}^{\circ}(s)
$$

The main result of this section states the properties of rescaled approximable quasistatic evolutions and will be proved at the end of this section, after a few technical steps.

Theorem 5.4. Let $\left(\Gamma^{\circ}, t^{\circ}\right)$ be a rescaled approximable quasistatic evolution as in Definition 5.1. Then, with the notation as above, the following hold:

(i) for every $x \in \mathrm{E}^{\circ}$ (see (5.10) for the definition of $\mathrm{E}^{\circ}$ ), there exists $J \subset[0, S]$ closed interval (which can also reduce to a singleton and contains $S$ if $x \in \mathrm{B}^{\circ}(S)$ ) such that

$$
x \in \mathrm{T}^{\circ}(s) \text { if and only if } s \in J
$$

(ii) if $n \in\{0, \ldots, N\}, j \in\left\{1, \ldots, h_{n}\right\}$, and $\left(s_{1}, s_{2}\right) \subset I_{n}^{\circ}$ are such that $\gamma_{j}^{\circ}(s) \notin \mathrm{E}^{\circ}$ for every $s \in\left(s_{1}, s_{2}\right)$, then

$$
\left(s_{1}, s_{2}\right) \ni s \mapsto \mathcal{G}\left(t^{\circ}(s) ; \Gamma^{\circ}(s), \gamma_{j}^{\circ}(s)\right) \quad \text { is continuous }
$$

and for a.e. $s \in\left(s_{1}, s_{2}\right)$

$$
\begin{aligned}
& \left(l_{j}^{\circ}\right)^{\prime}(s) \geq 0 ; \\
& \text { If }\left(t^{\circ}\right)^{\prime}(s)>0, \text { then } \mathcal{G}\left(t^{\circ}(s) ; \Gamma^{\circ}(s), \gamma_{j}^{\circ}(s)\right) \leq 1 ; \\
& \text { If } \mathcal{G}\left(t^{\circ}(s) ; \Gamma^{\circ}(s), \gamma_{j}^{\circ}(s)\right)<1, \text { then } l_{j}^{\circ} \text { is constant in a neighborhood of } s .
\end{aligned}
$$


(iii) for every $s \in[0, T]$ it holds the energy-dissipation balance

$$
\begin{aligned}
\mathcal{E}\left(t^{\circ}(s) ; \Gamma^{\circ}(s)\right)+l^{\circ}(s)= & \mathcal{E}\left(0 ; \Gamma_{0}\right)+l_{0}+\int_{0}^{s}\left\langle\nabla u^{\circ}(\tau), \nabla \dot{w}^{\circ}(\tau)\right\rangle \mathrm{d} \tau \\
& +\sum_{n=0}^{\bar{n}} \int_{I_{n}^{\circ} \cap U^{\circ}} \sum_{j=1}^{h_{n}}\left(l_{j}^{\circ}\right)^{\prime}(\tau)\left[\mathcal{G}\left(w^{\circ}(\tau) ; \Gamma^{\circ}(\tau), \gamma_{j}^{\circ}(\tau)\right)-1\right] \mathrm{d} \tau \\
& +\int_{\left(s^{n}, s\right) \cap U^{\circ}} \sum_{j=1}^{h_{\bar{n}}}\left(l_{j}^{\circ}\right)^{\prime}(\tau)\left[\mathcal{G}\left(w^{\circ}(\tau) ; \Gamma^{\circ}(\tau), \gamma_{j}^{\circ}(\tau)\right)-1\right] \mathrm{d} \tau,
\end{aligned}
$$

where $\bar{n}$ is such that $s \in I_{\bar{n}}^{\circ}$, and we adopt the convention $\left(l_{j}^{\circ}\right)^{\prime}(\tau) \mathcal{G}\left(w^{\circ}(\tau) ; \Gamma^{\circ}(\tau), \gamma_{j}^{\circ}(\tau)\right)=0$ if $\gamma_{j}^{\circ}(\tau) \notin \mathrm{G}_{\Gamma^{\circ}(\tau)}$; see (2.9) for the definition of $\mathrm{G}_{\Gamma^{\circ}(\tau)}$.

Remark 5.5. Assume that $\tau$ belongs to the interior part of $I_{n}^{\circ}$, so that $\gamma_{j}^{\circ}(\tau) \notin \mathrm{F}$. If $\gamma_{j}^{\circ}(\tau) \notin$ $\mathrm{G}_{\Gamma^{\circ}(\tau)}$, then, by Remark 5.3, we have $\gamma_{j}^{\circ}(\tau) \in \mathrm{B}^{\circ}(S)$. So the energy release rate is not defined at $\gamma_{j}^{\circ}(\tau)$, since there are no extensions of $\Gamma^{\circ}(\tau)$ at $\gamma_{j}^{\circ}(\tau)$ (see Definition 2.8), but the tip stops at $\gamma_{j}^{\circ}(\tau)$, and then the velocity is null. This justifies the convention $\left(l_{j}^{\circ}\right)^{\prime}(\tau) \mathcal{G}\left(w^{\circ}(\tau) ; \Gamma^{\circ}(\tau), \gamma_{j}^{\circ}(\tau)\right)=0$ for $\gamma_{j}^{\circ}(\tau) \notin \mathrm{G}_{\Gamma^{\circ}(\tau)}$.

Remark 5.6. Let us fix $\left(s_{1}, s_{2}\right) \subset I_{n}^{\circ}$ such that $\gamma_{j}^{\circ}(s) \notin \mathrm{E}^{\circ}$ for every $s \in\left(s_{1}, s_{2}\right)$. Assuming (5.12a) and $(5.12 \mathrm{~b})$, the condition $(5.12 \mathrm{c})$ implies that for a.e. $s \in\left(s_{1}, s_{2}\right)$ the following hold:

$$
\begin{aligned}
& \text { If }\left(t^{\circ}\right)^{\prime}(s)>0 \text { and }\left(l_{j}^{\circ}\right)^{\prime}>0, \text { then } \mathcal{G}\left(t^{\circ}(s) ; \Gamma^{\circ}(s), \gamma_{j}^{\circ}(s)\right)=1 ; \\
& \text { If }\left(t^{\circ}\right)^{\prime}(s)=0 \text { and }\left(l_{j}^{\circ}\right)^{\prime}>0, \text { then } \mathcal{G}\left(t^{\circ}(s) ; \Gamma^{\circ}(s), \gamma_{j}^{\circ}(s)\right) \geq 1 .
\end{aligned}
$$

In particular, in view of $(5.11)$,

$$
\text { if } \mathcal{G}\left(t^{\circ}(s), \Gamma^{\circ}(s), \gamma_{j}^{\circ}(s)\right)>1 \text {, then } s \in U^{\circ} .
$$

(See (5.8) for the definition of $U^{\circ}$.)

In order to prove Theorem 5.4, we employ the result below, which follows the lines of Proposition 4.10. There a crucial point was to use the fact that the discrete lengths $\underline{l}_{\varepsilon, k}$ were equi- $H^{1}$ for $\varepsilon$ fixed. In the current setting, the lengths $l_{\varepsilon}^{\circ}$ are equi-Lipschitz with respect to $\varepsilon$.

Proposition 5.7. Let $\tilde{s} \in I_{n}^{\circ}$ such that $\gamma_{j}^{\circ}(\tilde{s}) \notin \mathrm{F}$, and let

$$
r_{j}^{\circ}(\tilde{s}):=\omega\left(\mathrm{d}\left(\gamma_{j}^{\circ}(\tilde{s}), \mathrm{F}\right)\right) \wedge \eta \quad \text { and } \quad \bar{s}_{j}^{\circ}(\tilde{s}):=\left[\tilde{s}-\frac{r_{j}^{\circ}(\tilde{s})}{2}\right] \vee s^{n},
$$

where $\omega$ is the modulus of continuity introduced in Remark 2.3. Then there exists $\varepsilon_{0}>0$ such that for every $\varepsilon \in\left(0, \varepsilon_{0}\right)$ and $s \in\left(\bar{s}_{j}^{\circ}(\tilde{s}), \tilde{s}\right]$ the following hold:

$$
\begin{aligned}
& \mathrm{T}_{\varepsilon}^{\circ}(s) \cap \overline{B_{r_{j}^{\circ}(\tilde{s})}\left(\gamma_{j}^{\circ}(\tilde{s})\right)} \text { contains one and only one element, called } p_{\varepsilon}^{j}(s), \\
& \mathrm{S}_{\varepsilon}^{\circ}(s) \cap \overline{B_{r_{j}^{\circ}(\tilde{s})}\left(\gamma_{j}^{\circ}(\tilde{s})\right)}=\emptyset \\
& \Gamma_{\varepsilon}^{\circ}(s) \cap \overline{B_{r_{j}^{\circ}(\tilde{s})}\left(\gamma_{j}^{\circ}(\tilde{s})\right)} \in \mathcal{R}_{\eta} .
\end{aligned}
$$

Proof. Arguing as in the proof of Proposition 4.10 it is possible to prove that (5.15) holds for $s=\tilde{s}$ and that there exists $\varepsilon_{0}>0$ such that for $\varepsilon \in\left(0, \varepsilon_{0}\right)$

$$
\mathcal{H}^{1}\left(\Gamma_{\varepsilon}^{\circ}(\tilde{s}) \cap \overline{B_{r_{j}^{\circ}(\tilde{s})}\left(\gamma_{j}^{\circ}(\tilde{s})\right)}\right) \geq \frac{r_{j}^{\circ}(\tilde{s})}{2} .
$$

In order to see the corresponding properties for general $s$, it is enough to show that, for

$$
s_{\varepsilon}:=\min \left\{s \in\left[s^{n}, \tilde{s}\right): \Gamma_{\varepsilon}^{\circ}(s) \cap \overline{B_{r_{j}^{\circ}(\tilde{s})}\left(\gamma_{j}^{\circ}(\tilde{s})\right)} \neq \emptyset\right\},
$$

it holds $s_{\varepsilon} \leq \bar{s}_{j}^{\circ}(\tilde{s})$ for $\varepsilon \in\left(0, \varepsilon_{0}\right)$. This is implied by (5.6), which gives

$$
\frac{r}{2} \leq \mathcal{H}^{1}\left(\Gamma_{\varepsilon}^{\circ}(\tilde{s}) \backslash \Gamma_{\varepsilon}^{\circ}\left(s_{\varepsilon}\right)\right)=\int_{s_{\varepsilon}}^{\tilde{s}}\left(l_{\varepsilon}^{\circ}\right)^{\prime}(s) \mathrm{d} s \leq \tilde{s}-s_{\varepsilon} .
$$

and concludes the proof. 
Proof of Theorem 5.4. Arguing as done in Theorem 4.7, we can prove the statement (i) and the fact that $\left(s^{n}, s^{n+1}\right)$ is the union of a finite number of open intervals where $\gamma_{j}^{\circ}(s) \notin \mathrm{E}^{\circ}$, and of a finite number of closed intervals in each of which $\gamma_{j}^{\circ}(s)$ is constant and belongs to $\mathrm{E}^{\circ}$.

In order to show (ii), let us fix $n \in\{0, \ldots, N\}, j \in\left\{1, \ldots, h_{n}\right\}$, and $\left(s_{1}, s_{2}\right) \subset I_{n}^{\circ}$ such that $\gamma_{j}^{\circ}(s) \notin \mathrm{E}^{\circ}$ for $s \in\left(s_{1}, s_{2}\right)$. As in Proposition 4.14, by Propositions 2.12 and 5.7 we deduce (5.11) and the convergence

$$
\mathcal{G}\left(t_{\varepsilon}^{\circ}(s) ; \Gamma_{\varepsilon}^{\circ}(s), p_{\varepsilon}^{j}(s)\right) \rightarrow \mathcal{G}\left(t^{\circ}(s) ; \Gamma^{\circ}(s), \gamma_{j}^{\circ}(s)\right) \text { for every } s \in\left(s_{1}, s_{2}\right) .
$$

By (5.6), the functions

$$
s \in\left(\bar{s}_{j}^{\circ}(\tilde{s}), \tilde{s}\right] \mapsto\left(g_{\varepsilon}^{\circ}\right)^{j}(s):=\mathcal{H}^{1}\left(\Gamma_{\varepsilon}^{\circ}(s) \cap B_{r_{j}^{\circ}(\tilde{s})}\left(\gamma_{j}^{\circ}(\bar{s})\right)\right)
$$

are 1-Lipschitz and, by (5.3a),

$$
\left(g_{\varepsilon}^{\circ}\right)_{j} \stackrel{*}{\rightarrow}\left(g^{\circ}\right)_{j} \quad \text { in } W^{1, \infty}\left(\left(\bar{s}_{j}^{\circ}(\tilde{s}), \tilde{s}\right]\right),
$$

where

$$
s \in\left(\bar{s}_{j}^{\circ}(\tilde{s}), \tilde{s}\right] \mapsto\left(g^{\circ}\right)_{j}(s):=\mathcal{H}^{1}\left(\Gamma^{\circ}(s) \cap B_{r_{j}^{\circ}(\tilde{s})}\left(\gamma_{j}^{\circ}(\bar{s})\right)\right)=l_{j}^{\circ}(s)-\mathcal{H}^{1}\left(\gamma_{j}^{\circ}\left(\left[s^{n}, s\right]\right) \backslash B_{r_{j}^{\circ}(\tilde{s})}\left(\gamma_{j}^{\circ}(\bar{s})\right)\right) .
$$

Notice that the time derivative of $\left(g_{\varepsilon}^{\circ}\right)_{j}$ depends only on $\Gamma_{\varepsilon}^{\circ}, s$, and on $p_{\varepsilon}^{j}(s)$. Thus we define

$$
v_{\varepsilon}^{\circ}\left(s, p_{\varepsilon}^{j}(s)\right):=\left(g_{\varepsilon}^{\circ}\right)_{j}^{\prime}(s) .
$$

We also observe that the time interval $I_{n}^{\circ}$ may be approximated e.g. by two different intervals $I_{\varepsilon}^{n_{1}}$, $I_{\varepsilon}^{n_{2}}$ : this is due to the fact that a branch of $\Gamma_{\varepsilon}(T)$ may disappear in the limit as $\varepsilon \rightarrow 0$. For this reason we will have $p_{\varepsilon}^{j}(s)=\gamma_{\varepsilon}^{i}\left(l_{\varepsilon}^{i}\left(t_{\varepsilon}^{\circ}(s)\right)\right)$ for some $i$ possibly depending on $\varepsilon$ and $s$. (See (4.3) for the definition of $\left.l_{\varepsilon}^{i}\right)$. In particular,

$$
v_{\varepsilon}^{\circ}\left(s, p_{\varepsilon}^{j}(s)\right)=i_{\varepsilon}^{i}\left(t_{\varepsilon}^{\circ}(s)\right)\left(t_{\varepsilon}^{\circ}\right)^{\prime}(s) .
$$

As in the previous section (see (4.18)), for $\left(\bar{s}_{1}, \bar{s}_{2}\right) \subset \subset I_{n}^{\circ}$ such that $\gamma_{j}^{\circ}(s) \notin \mathrm{F}$ for every $s \in\left(\bar{s}_{1}, \bar{s}_{2}\right)$, we have that

$$
v_{\varepsilon}^{\circ}\left(\cdot, p_{\varepsilon}^{j}(\cdot)\right) \stackrel{*}{\rightarrow}\left(l_{j}^{\circ}\right)^{\prime} \quad \text { in } L^{\infty}\left(\bar{s}_{1}, \bar{s}_{2}\right) .
$$

By $(5.17)$ and the fact that $0<\left(t_{\varepsilon}^{\circ}\right)^{\prime}(s)$, we can rewrite (4.20) in the new variables as

$$
\begin{aligned}
& v_{\varepsilon}^{\circ}\left(s, p_{\varepsilon}^{j}(s)\right) \geq 0, \\
& \left(t_{\varepsilon}^{\circ}\right)^{\prime}(s)-\mathcal{G}\left(t_{\varepsilon}^{\circ}(s) ; \Gamma_{\varepsilon}^{\circ}(s), p_{\varepsilon}^{j}(s)\right)\left(t_{\varepsilon}^{\circ}\right)^{\prime}(s)+\varepsilon v_{\varepsilon}^{\circ}\left(s, p_{\varepsilon}^{j}(s)\right) \geq 0, \\
& {\left[\left(t_{\varepsilon}^{\circ}\right)^{\prime}(s)-\mathcal{G}\left(t_{\varepsilon}^{\circ}(s) ; \Gamma_{\varepsilon}^{\circ}(s), p_{\varepsilon}^{j}(s)\right)\left(t_{\varepsilon}^{\circ}\right)^{\prime}(s)+\varepsilon v_{\varepsilon}^{\circ}\left(s, p_{\varepsilon}^{j}(s)\right)\right] v_{\varepsilon}^{\circ}\left(s, p_{\varepsilon}^{j}(s)\right)=0,}
\end{aligned}
$$

for a.e. $s \in\left(s_{1}, s_{2}\right)$.

As in Lemma 4.12, for every $s \in\left(s_{1}, s_{2}\right)$ there exists a set $A_{j}^{\circ}(s) \subset\left(s_{1}, s_{2}\right)$, at most countable, such that $\left(\bar{s}_{j}^{\circ}\left(t_{1}\right), t_{1}\right]$ and $\left(\bar{s}_{j}^{\circ}\left(t_{2}\right), t_{2}\right]$ are disjoint for $t_{1} \neq t_{2} \in A_{j}^{\circ}(s)$ and

$$
\left(s_{1}, s\right]=\bigcup_{\tilde{s} \in A_{j}^{\circ}(s)}\left(\bar{s}_{j}^{\circ}(\tilde{s}), \tilde{s}\right] .
$$

Let us fix a subinterval $\left(\bar{s}_{j}^{\circ}(\tilde{s}), \tilde{s}\right] \subset\left(s_{1}, s_{2}\right)$. By Proposition 5.7, there exists $\varepsilon_{0}$ such that $(5.15)$ holds for $\varepsilon \geq \varepsilon_{0}$ and $s \in\left(\bar{s}_{j}^{\circ}(\tilde{s}), \tilde{s}\right]$. Arguing as in the proof of Proposition 4.14, we get that there exists a positive constant $C$ independent of $\varepsilon \geq \varepsilon_{0}$ and $s \in\left(\bar{s}_{j}^{\circ}(\tilde{s}), \tilde{s}\right]$ such that

$$
\mathcal{G}\left(t_{\varepsilon}^{\circ}(s) ; \Gamma_{\varepsilon}^{\circ}(s), p_{\varepsilon}^{j}(s)\right) \leq C \quad \text { for every } \varepsilon \geq \varepsilon_{0} \text { and } s \in\left(\bar{s}_{j}^{\circ}(\tilde{s}), \tilde{s}\right] .
$$

Employing the fact that $\mathcal{G}\left(t_{\varepsilon}^{\circ}(s) ; \Gamma_{\varepsilon}^{\circ}(s), p_{\varepsilon}^{j}(s)\right) \geq 0$, and (5.16), we have that

$$
\mathcal{G}\left(t_{\varepsilon}^{\circ}(\cdot) ; \Gamma_{\varepsilon}^{\circ}(\cdot), p_{\varepsilon}^{j}(\cdot)\right) \rightarrow \mathcal{G}\left(t^{\circ}(\cdot) ; \Gamma^{\circ}(\cdot), \gamma_{j}^{\circ}(\cdot)\right) \quad \text { in } L^{q}\left(\bar{s}_{j}^{\circ}(\tilde{s}), \tilde{s}\right), \quad \text { for every } q \in[1,+\infty) \text {. }
$$

Let $\varphi \in L^{2}\left(\bar{s}_{j}^{\circ}(\tilde{s}), \tilde{s}\right)$ such that $\varphi \geq 0$. By $(5.19 \mathrm{~b})$

$$
\int_{\bar{s}_{j}^{\circ}(\tilde{s})}^{\tilde{s}} \varphi(s)\left[\left(t_{\varepsilon}^{\circ}\right)^{\prime}(s)-\mathcal{G}\left(t_{\varepsilon}^{\circ}(s) ; \Gamma_{\varepsilon}^{\circ}(s), p_{\varepsilon}^{j}(s)\right)\left(t_{\varepsilon}^{\circ}\right)^{\prime}(s)+\varepsilon v_{\varepsilon}^{\circ}\left(s, p_{\varepsilon}^{j}(s)\right)\right] \mathrm{d} s \geq 0 .
$$

By (5.3b), (5.18), and (5.20) we can pass to the limit obtaining that

$$
\int_{\bar{s}_{j}^{\circ}(\tilde{s})}^{\tilde{s}} \varphi(s)\left[1-\mathcal{G}\left(t^{\circ}(s) ; \Gamma^{\circ}(s), \gamma_{j}^{\circ}(s)\right)\right]\left(t^{\circ}\right)^{\prime}(s) \mathrm{d} s \geq 0,
$$


and then $(5.12 \mathrm{~b})$ follows by the arbitrariness of $\varphi$ and $\tilde{s}$.

Let us prove $(5.12 \mathrm{c})$. First we show that, if $\mathcal{G}\left(t^{\circ}(s) ; \Gamma^{\circ}(s), \gamma_{j}^{\circ}(s)\right)<1$, then there exists $\delta>0$, depending only on $s$, such that

$$
\mathcal{G}\left(t_{\varepsilon_{k}}^{\circ}(\cdot) ; \Gamma_{\varepsilon_{k}}^{\circ}(\cdot), p_{\varepsilon_{k}}^{j}(\cdot)\right)<1 \quad \text { in }(s-\delta, s+\delta)
$$

for $k$ sufficiently large. Otherwise, assume that there exist a sequence $t_{k} \rightarrow s$ such that

$$
\mathcal{G}\left(t_{\varepsilon_{k}}^{\circ}\left(t_{k}\right) ; \Gamma_{\varepsilon_{k}}^{\circ}\left(t_{k}\right), p_{\varepsilon_{k}}^{j}\left(t_{k}\right)\right) \geq 1
$$

Since $\left|t_{\varepsilon}^{\circ}\left(t_{k}\right)-t_{\varepsilon}^{\circ}(s)\right| \leq\left|t_{k}-s\right|$ and $\left|l_{\varepsilon}^{\circ}\left(t_{k}\right)-l_{\varepsilon}^{\circ}(s)\right| \leq\left|t_{k}-s\right|$ for every $\varepsilon>0$, we are allowed to apply Proposition 2.12. It follows that $\mathcal{G}\left(t^{\circ}(s) ; \Gamma^{\circ}(s), \gamma_{j}^{\circ}(s)\right) \geq 1$, which contradicts (5.22). Now, by $(5.19 \mathrm{c})$ and $(5.21)$, we get that $p_{\varepsilon_{k}}^{j}$ is constant in $(s-\delta, s+\delta)$ and so is $l_{j}^{\circ}$. Then (ii) is proved.

Let us now show (iii). Arguing as in Proposition 4.9 we have that the total energy $\mathcal{F}\left(t^{\circ}(\cdot) ; \Gamma^{\circ}(\cdot)\right)$ is $A C_{\text {loc }}\left(I_{n}^{\circ}\right)$ for every $n \in\{0, \ldots, N\}$ and that for a.e. $s \in I_{n}^{\circ}=\left(s^{n}, s^{n+1}\right]$

$$
\frac{\mathrm{d}}{\mathrm{d} s} \mathcal{F}\left(t^{\circ}(s) ; \Gamma^{\circ}(s)\right)=\sum_{j=1}^{h_{n}}\left[1-\mathcal{G}\left(t^{\circ}(s) ; \Gamma^{\circ}(s), \gamma_{j}^{\circ}(s)\right)\right]\left(l_{j}^{\circ}\right)^{\prime}(s)+\left\langle\nabla u^{\circ}(s), \nabla \dot{w}^{\circ}(s)\right\rangle,
$$

with the convention $\mathcal{G}\left(t^{\circ}(s) ; \Gamma^{\circ}(s), \gamma_{j}^{\circ}(s)\right)\left(l_{j}^{\circ}\right)^{\prime}(s)=0$ if $\gamma_{j}^{\circ}(s) \notin \mathrm{G}_{\Gamma^{\circ}(\tau)}$.

Integrating in $\left(s_{1}, s_{2}\right) \subset \subset I_{n}^{\circ}$,

$$
\begin{aligned}
\mathcal{F}\left(t^{\circ}\left(s_{2}\right) ; \Gamma^{\circ}\left(s_{2}\right)\right)-\mathcal{F}\left(t^{\circ}\left(s_{1}\right) ; \Gamma^{\circ}\left(s_{1}\right)\right)= & \int_{s_{1}}^{s_{2}} \sum_{j=1}^{h_{n}}\left[1-\mathcal{G}\left(t^{\circ}(\tau) ; \Gamma^{\circ}(\tau), \gamma_{j}^{\circ}(\tau)\right)\right]\left(l_{j}^{\circ}\right)^{\prime}(\tau) \mathrm{d} \tau \\
& +\int_{s_{1}}^{s_{2}}\left\langle\nabla u^{\circ}(\tau), \nabla \dot{w}^{\circ}(\tau)\right\rangle \mathrm{d} \tau .
\end{aligned}
$$

Notice that we can pass to the limit as $s_{1} \rightarrow s^{n}$ and $s_{2} \rightarrow s^{n+1}$ since the positive part of $\sum_{j=1}^{h_{n}}\left[1-\mathcal{G}\left(t^{\circ}(\tau) ; \Gamma^{\circ}(\tau), \gamma_{j}^{\circ}(\tau)\right)\right]\left(l_{j}^{\circ}\right)^{\prime}(\tau)$ is less than one and we can use Monotone Convergence Theorem for the negative part. Since $\mathcal{F}\left(t^{\circ}(\cdot) ; \Gamma^{\circ}(\cdot)\right)$ is continuous, we can then sum up over the intervals $I_{n}^{\circ}$, whose number is finite.

We are left to prove that in the last two lines of (5.13) there is no contribution for $\tau \notin U^{\circ}$. As observed before, $\left(s^{n}, s^{n+1}\right)$ is the union of a finite number of open intervals such that $\gamma_{j}^{\circ}(s) \notin \mathrm{E}^{\circ}$ for every $s$ in these subintervals, and of a finite number of closed intervals in each of which $\gamma_{j}^{\circ}$ is constant and belongs to $\mathrm{E}^{\circ}$. If we are in an interval of the first type, by (5.12c) and (5.14),

$$
\sum_{j=1}^{h_{n}}\left[1-\mathcal{G}\left(t^{\circ}(s) ; \Gamma^{\circ}(s), \gamma_{j}^{\circ}(s)\right)\right]\left(l_{j}^{\circ}\right)^{\prime}(s)=0 \quad \text { for } s \notin U^{\circ} .
$$

On the other hand, if we are in an interval $\left[\bar{s}_{1}, \bar{s}_{2}\right]$ such that $\gamma_{j}^{\circ}(s)=\bar{x} \in \mathrm{E}^{\circ}$, then $\left(l_{j}^{\circ}\right)^{\prime}(s)=0$. (Recall also the convention adopted for the points $\bar{x} \notin \mathrm{G}_{\Gamma^{\circ}(s)}$.) Threfore we conclude (5.13) and the proof is completed.

Remark 5.8. Arguing as in [19, Theorem 8.7] we have that for every $n \in\{0, \ldots, N\}, j \in$ $\left\{1, \ldots, h_{n}\right\}$, and $\left(s_{1}, s_{2}\right) \subset I_{n}^{\circ}$ such that $\gamma_{j}^{\circ}(s) \notin \mathrm{E}^{\circ}$ for every $s \in\left(s_{1}, s_{2}\right)$, there exists a continuous function $\lambda:\left(s_{1}, s_{2}\right) \rightarrow[0,+\infty)$, independent of $j$, such that for a.e. $s$ and every $j$

$$
\lambda(s)\left(l_{j}^{\circ}\right)^{\prime}(s)=\left(\mathcal{G}\left(t^{\circ}(s) ; \Gamma^{\circ}(s), \gamma_{j}^{\circ}(s)\right)-1\right)^{+} \quad \text { and } \quad \lambda(s)\left(t^{\circ}\right)^{\prime}(s)=0 .
$$

Therefore, the rescaled evolution is governed by a viscous law in $U^{\circ}$. This gives insight on the unstable propagations, which correspond to jumps regime in the original time scale.

Acknowledgments. The authors thank Gianni Dal Maso for useful discussions. This work has been partially supported by the European Research Council through the Advanced Grant No. 290888 Quasistatic and Dynamic Evolution Problems in Plasticity and Fracture and by the GNAMPA (Gruppo Nazionale per l'Analisi Matematica, la Probabilità e le loro Applicazioni) of INdAM (Istituto Nazionale di Alta Matematica) through the 2016 Project Analisi multiscala di sistemi complessi con metodi variazionali. 


\section{REFERENCES}

[1] J.-F. Babadjian, A. Chambolle, and A. Lemenant, Energy release rate for non-smooth cracks in planar elasticity, J. Éc. polytech. Math., 2 (2015), pp. 117-152.

[2] B. Bourdin, G. A. Francfort, and J.-J. Marigo, The variational approach to fracture, J. Elasticity, 91 (2008), pp. 5-148.

[3] A. Chambolle, A density result in two-dimensional linearized elasticity, and applications, Arch. Ration. Mech. Anal., 167 (2003), pp. 211-233.

[4] A. Chambolle, G. A. Francfort, and J.-J. Marigo, Revisiting energy release rates in brittle fracture, J. Nonlinear Sci., 20 (2010), pp. 395-424.

[5] A. Chambolle and A. Lemenant, The stress intensity factor for non-smooth fractures in antiplane elasticity, Calc. Var. Partial Differential Equations, 47 (2013), pp. 589-610.

[6] V. CRismale and G. Lazzaroni, Viscous approximation of quasistatic evolutions for a coupled elastoplasticdamage model, Calc. Var. Partial Differential Equations, 55 (2016), pp. 54, Art. 17.

[7] G. Dal Maso, A. DeSimone, and F. Solombrino, Quasistatic evolution for Cam-Clay plasticity: a weak formulation via viscoplastic regularization and time rescaling, Calc. Var. Partial Differ. Equ., 40 (2011), pp. 125181.

[8] G. Dal Maso, G. A. Francfort, and R. Toader, Quasistatic crack growth in nonlinear elasticity, Arch. Ration. Mech. Anal., 176 (2005), pp. 165-225.

[9] G. Dal Maso and G. Lazzaroni, Quasistatic crack growth in finite elasticity with non-interpenetration, Ann. Inst. H. Poincaré Anal. Non Linéaire, 27 (2010), pp. 257-290.

[10] G. Dal Maso and R. Toader, A model for the quasi-static growth of brittle fractures: existence and approximation results, Arch. Ration. Mech. Anal., 162 (2002), pp. 101-135.

[11] M. A. Efendiev And A. Mielke, On the rate-independent limit of systems with dry friction and small viscosity, J. Convex Anal., 13 (2006), pp. 151-167.

[12] G. A. Francfort and C. J. LARSEn, Existence and convergence for quasi-static evolution in brittle fracture, Comm. Pure Appl. Math., 56 (2003), pp. 1465-1500.

[13] G. A. Francfort and J.-J. Marigo, Revisiting brittle fracture as an energy minimization problem, J. Mech. Phys. Solids, 46 (1998), pp. 1319-1342.

[14] A. A. Griffith, The phenomena of rupture and flow in solids, Philos. Trans. Roy. Soc. London Ser. A, 221 (1920), pp. 163-198.

[15] D. Knees, A. Mielke, And C. Zanini, On the inviscid limit of a model for crack propagation, Math. Models Methods Appl. Sci., 18 (2008), pp. 1529-1569.

[16] D. Knees, R. Rossi, And C. Zanini, A vanishing viscosity approach to a rate-independent damage model, Math. Models Methods Appl. Sci., 23 (2013), pp. 565-616.

[17] D. Knees, C. Zanini, And A. Mielke, Crack growth in polyconvex materials, Phys. D, 239 (2010), pp. 14701484.

[18] G. Lazzaroni and R. Toader, Energy release rate and stress intensity factor in antiplane elasticity, J. Math. Pures Appl. (9), 95 (2011), pp. 565-584.

[19] — A model for crack propagation based on viscous approximation, Math. Models Methods Appl. Sci., 21 (2011), pp. 2019-2047.

[20] A. Mielke, R. Rossi, and G. Savaré, BV solutions and viscosity approximations of rate-independent systems, ESAIM Control Optim. Calc. Var., 18 (2012), pp. 36-80.

$[21] \longrightarrow$, Balanced viscosity (BV) solutions to infinite-dimensional rate-independent systems, J. Eur. Math. Soc. (JEMS), 18 (2016), pp. 2107-2165.

[22] — Balanced-viscosity solutions for multi-rate systems, Journal of Physics: Conference Series, 727 (2016), pp. 26, Art. 012010.

[23] A. Mielke And T. RoubíčEK, Rate-independent systems, vol. 193 of Applied Mathematical Sciences, Springer, New York, 2015. Theory and application.

[24] M. NeGRI, A comparative analysis on variational models for quasi-static brittle crack propagation, Adv. Calc. Var., 3 (2010), pp. 149-212.

[25] M. NeGRI, Energy release rate along a kinked path, Math. Methods Appl. Sci., 34 (2011), pp. 384-396.

[26] M. Negri And C. Ortner, Quasi-static crack propagation by Griffith's criterion, Math. Models Methods Appl. Sci., 18 (2008), pp. 1895-1925.

[27] S. RACCA, A model for crack growth with branching and kinking, Asymptot. Anal., 89 (2014), pp. 63-110.

SisSA, via Bonomea 265, 34136 Trieste, Italy

E-mail address, V. Crismale: vito.crismale@sissa.it

E-mail address, G. Lazzaroni: giuliano.lazzaroni@sissa.it 